Historical perspective

\title{
Structure and energy of liquid foams
}

\author{
Wiebke Drenckhan ${ }^{\mathrm{a}, *}$, Stefan Hutzler ${ }^{\mathrm{b}}$ \\ a Laboratoire de Physique des Solides, Université de Paris-Sud, CNRS UMR 8502, Orsay, France \\ b School of Physics, Trinity College Dublin, Ireland
}

\section{A R T I C L E I N F O}

Available online $\mathrm{xxxx}$

\section{Keywords:}

Foam structure

Emulsion structure

Osmotic pressure

Interfacial energy

Jamming

\begin{abstract}
A B S T R A C T
We present an overview of recent advances in the understanding of foam structure and energy and their dependence on liquid volume fraction. We consider liquid foams in equilibrium for which the relevant energy is surface energy. Measurements of osmotic pressure can be used to determine this as a function of liquid fraction in good agreement with results from computer simulations. This approach is particularly useful in the description of foams with high liquid content, so-called wet foams. For such foams X-ray tomography proves to be an important technique in analysing order and disorder. Much of the discussion in this article is also relevant to bi-liquid foams, i.e. emulsions, and to solid foams, provided that the solidification preserves the structure of the initially liquid foam template.
\end{abstract}

(c) 2015 Elsevier B.V. All rights reserved.

\section{Contents}

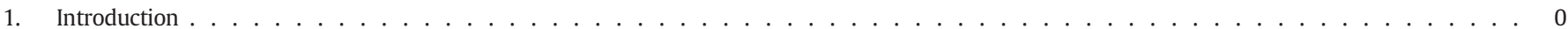

2. The dry limit $(\varphi<005) \ldots \ldots \ldots$

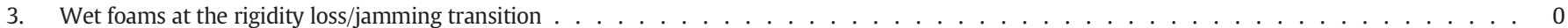

4. Interpolating between the limits of wet and dry foams . . . . . . . . . . . . . . . . . . . . . . . . . . . . . . . . . . . . 0

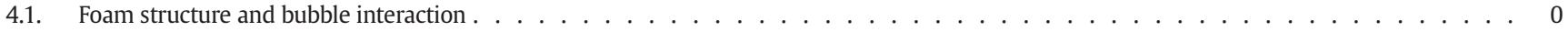

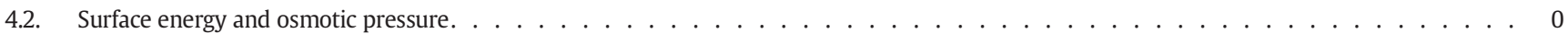

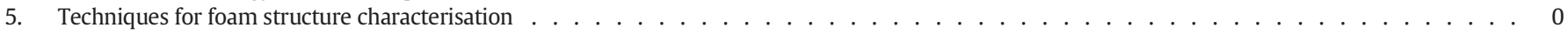

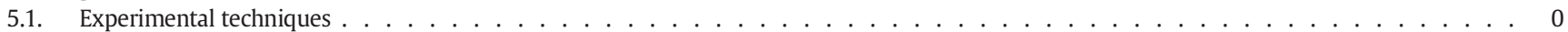

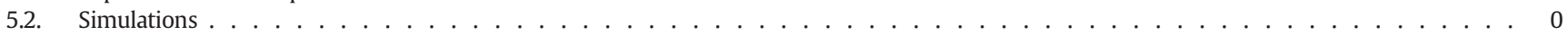

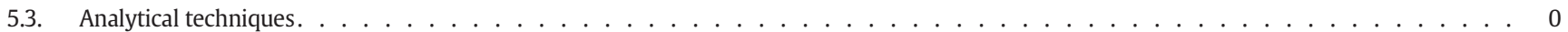

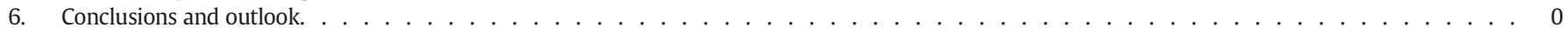

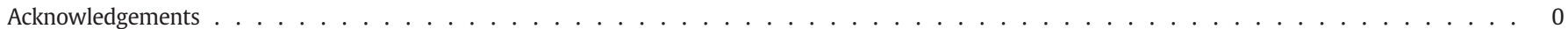

Appendix A. Some structural relations for the Kelvin foam . . . . . . . . . . . . . . . . . . . . . . . . . 0

Appendix B. Osmotic pressure and profiles of liquid fraction . . . . . . . . . . . . . . . . . . . . . . . . . . . . . . . . 0

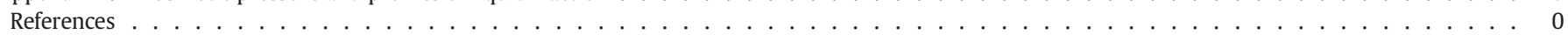

\section{Introduction}

Many properties of liquid foams are a direct consequence of their detailed geometrical structure. The opacity of foam samples, for example, is due to the diffusive light scattering from both liquid films and their intersections (Plateau borders). Foam drainage under gravity propagates mainly via the flow of liquid through the network of Plateau borders.

\footnotetext{
* Corresponding author.

E-mail address: Wiebke.Drenckhan@u-psud.fr (W. Drenckhan).
}

Also the electrical conductivity of a foam is determined by this network. The complex flow behaviour of foams under shear is due to the requirement of structural rearrangements. In this section we present a general overview of foam structure and introduce several quantities for its description.

In equilibrium, foam structure is determined by the minimisation of surface energy for a given volume fraction of liquid. When considering foams under gravity, this corresponds to the experimental situation where a freshly formed foam has been allowed to drain so that an equilibrium profile of liquid content is established - as can be seen in Fig. 1. Furthermore, the time scale of observation needs to be short enough to make foam ageing effects - such as volume changes due to gas diffusion 


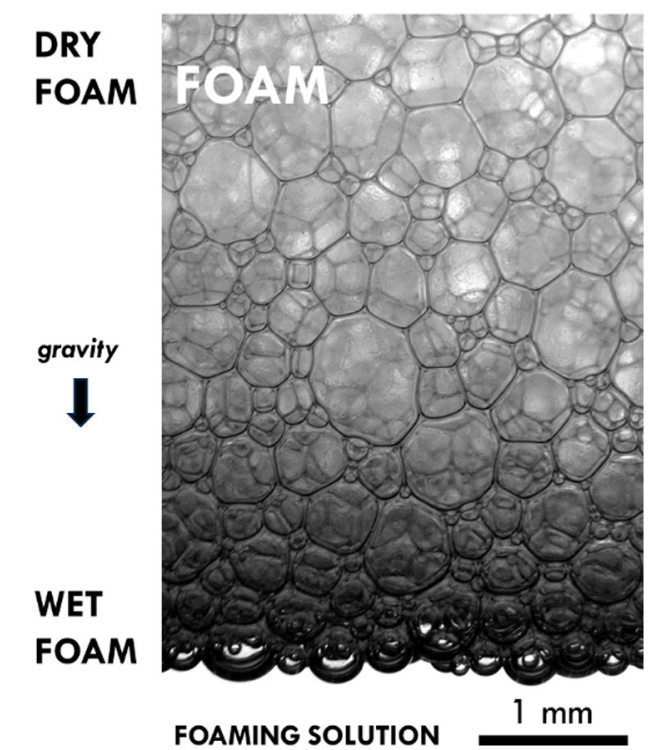

Fig. 1. Example of a liquid foam floating on top of its foaming solution (in this case the detergent "Fairy Liquid"). Liquid drainage under gravity results in a gradient of liquid fraction. At the top (dry foam) the bubbles take on polyhedral shapes while at the bottom (wet foam) they are nearly spherical.

between neighbouring bubbles (coarsening) or breaking films (coalescence) - negligible. Surface tension may then be treated as constant throughout the foam.

The excess pressure of a bubble inside a liquid with surface tension $\gamma$ and radius $R$ is given by the Young-Laplace law as $\Delta \mathrm{P}=2 \gamma / \mathrm{R}$. For a typical bubble of $\mathrm{R} \approx 100 \mu \mathrm{m}$ and $\gamma \approx 0.03 \mathrm{~N} / \mathrm{m}$, this leads to a pressure difference of the order of $1000 \mathrm{~Pa}$. This is sufficiently small in comparison with the atmospheric pressure of $10^{5} \mathrm{~Pa}$, which is why bubbles in foams may be considered as incompressible.

The energy $E$ of a foam with $n$ bubbles is then simply

$E=\gamma \sum_{i=1}^{n} S_{i}=\gamma S_{\text {tot }}$,

where $S_{\mathrm{i}}$ is the surface area of bubble " $i$ " and $S_{\text {tot }}$ is the total surface area of the foam.

The surface energy of the $100 \mu \mathrm{m}$ bubble considered above is thus of the order of $10^{-8} \mathrm{~J}$, i.e. about $10^{13}$ times larger than the thermal energy $k T \sim 10^{-21} \mathrm{~J}$. Note that also its potential energy $\Delta \rho g R^{4}$ due to buoyancy is about $10^{9}$ times larger than $k T$. Thermal energies therefore play no role in the organisation of bubble packings. This can be different in the case of emulsions, where density differences, values of surface tension and droplet sizes are often much smaller so that thermal effects (such as Brownian motion) need to be taken into account.

A photograph of a typical liquid foam in equilibrium under gravity is shown in Fig. 1. It reveals that bubble shape is dependent on the value of the local liquid fraction $\varphi$ of the foam, i.e. the ratio of the liquid volume to the foam volume under consideration. Close to the foam-liquid interface, at the bottom of the foam column, the bubbles are well approximated by contacting, deformed spheres (Section 3). This is often referred to as the wet limit. For foams with modest polydispersity (i.e. containing different-sized bubbles), the maximum value of liquid fraction, or critical liquid fraction $\varphi_{C}$, is thus $\varphi_{C} \approx 0.36$, as obtained for random packings of (monodisperse) hard spheres (Section 3). Foams with liquid fraction higher than about 0.15 are referred to as wet foams. For decreasing values of liquid fraction, further away from the liquid interface, the bubbles are increasingly deformed, with curved films between them. Foams with $\varphi$ less than about 0.05 are called dry (Section 2), their bubbles being nearly polyhedral in shape. The setting of precise values is quite arbitrary (see Weaire and Hutzler [1]). However, it helps to distinguish between dry foams, whose cellular structure is still well described by Plateau's rules (Section 2), and wet foams (Section 3) which resemble aggregations of near spherical bubbles.

Considering such a column of foam in equilibrium, floating on an underlying liquid, the height of the wet foam layer may be estimated as $l_{\mathrm{c}}^{2} /$ $D$ for bubbles of diameter $D$. Here $l_{\mathrm{c}}$ is the capillary length given by $l_{\mathrm{c}}^{2}=$ $\gamma /(\Delta \rho g), g$ is the gravitational acceleration and $\Delta \rho$ is the liquid density. $A$ wet foam thus contains about

$\operatorname{Pri}=\left(\frac{l_{c}}{D}\right)^{2}$

layers of bubbles, where Pri has been called the dimensionless Princen number in honour of the foam pioneer Henry Princen (Chapter 1 in [2]). For a typical foaming solution, with say $l_{\mathrm{c}} \approx 1.6 \mathrm{~mm}$, it follows that the average bubble diameter should not exceed $500 \mu \mathrm{m}$ if one wants to produce wet foams of more than 10 layers of bubbles.

The example shown in Fig. 1 is that of a polydisperse, disordered foam, typical of foams found in nature and many industrial applications. Foams that are currently in vogue in research laboratories are monodisperse, i.e. contain equal-volume bubbles with polydispersity of less than about five percent in radius. Fig. 2 shows the example of such a foam floating on its foaming solution. As can be seen, these foams have the tendency to order [3-6], especially in the presence of templating boundaries (Section 3) or when confined in narrow tubes. A selection of experimental realisations of such ordered foams is shown in Fig. 3. In sufficiently wet monodisperse foams ordering might also occur spontaneously in the bulk, as discussed in Section 3.

The surface energy of a foam depends on bubble size, foam structure and liquid fraction. The dependence on bubble size can be removed by introducing the (dimensionless) scaled energy $\hat{E}$ as

$$
\hat{E}=\frac{\langle S\rangle}{\langle V\rangle^{2 / 3}}
$$

where $\langle V\rangle$ and $\langle S>$ are mean bubble volume and surface area in a foam, respectively. Hence, $\hat{E}$ is a measure of how much bubbles are deformed away from their ideal spherical shape. For finite values of liquid fraction it is more convenient to introduce the relative surface excess $\varepsilon(\varphi)$ as

$\varepsilon(\varphi)=\frac{\langle S(\varphi)\rangle-\left\langle S_{0}\right\rangle}{\left\langle S_{0}\right\rangle}$

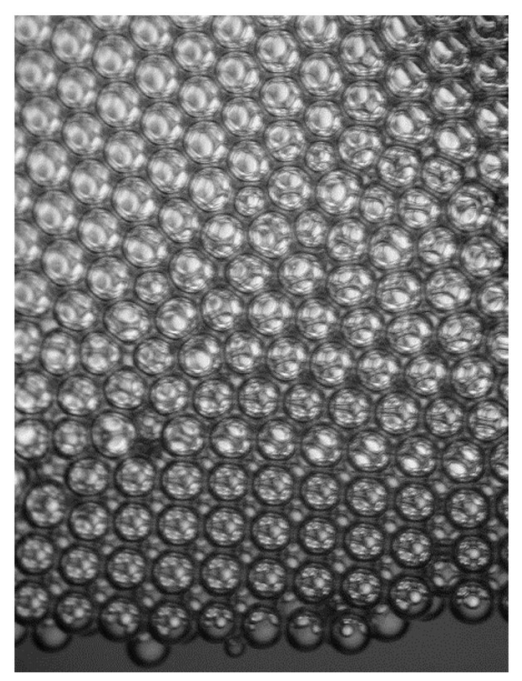

Fig. 2. Example of a monodisperse foam, floating on its foaming solution. It demonstrates the tendency of equal-volume bubbles to order (bubble size $\sim 500 \mu \mathrm{m}$, see also [7]). 
a

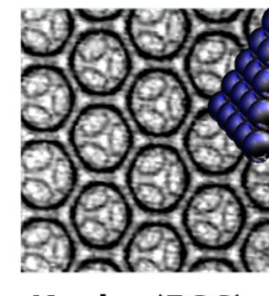

Kepler (FCC)

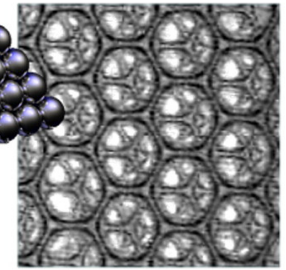

$\longleftarrow$ Liquid fraction $\varphi$

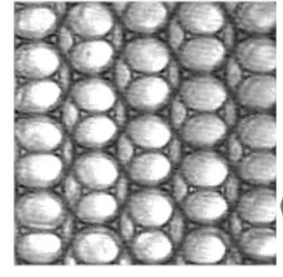

$\varphi$

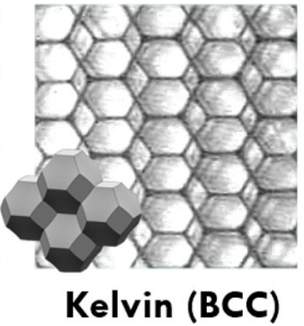

Kelvin (BCC) b

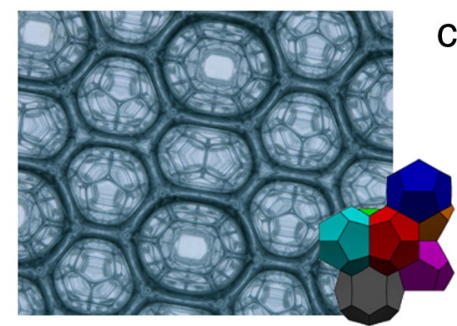

Weaire-Phelan foam
C

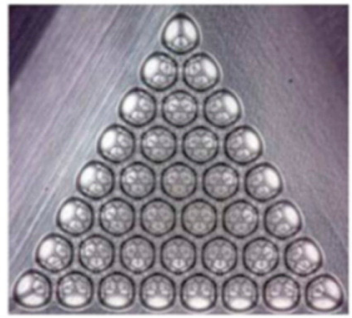

FCC

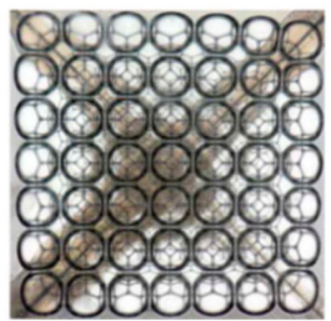

BCC

d
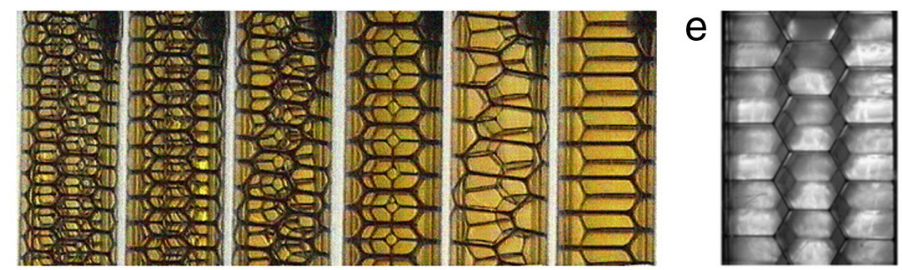

Ordered foams under confinement

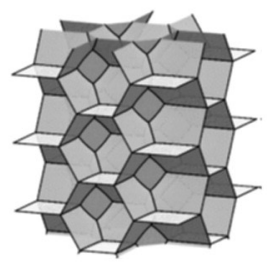

f

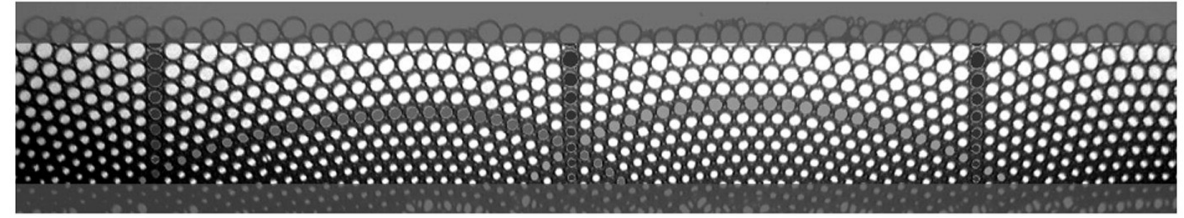

\section{Conformal foams}

Fig. 3. Experimental realisations of various periodic structures of liquid foams which self-order under the influence of gravity and/or confinement. All examples use monodisperse bubbles with bubble diameters between 0.5 and $1.5 \mathrm{~mm}$. (a) A hexagonally close-packed Kepler (FCC) packing of spherical bubbles transforms into a Kelvin foam (BCC) upon removal of liquid (reprinted with permission from [6], Copyright 2008, American Chemical Society). (b) A Weaire-Phelan foam contains two types of bubble shapes in the unit cell and is conjectured to be the structure of the lowest surface area for dry foams with equal-volume bubbles (from [8], reprinted by permission of the publisher Taylor \& Francis Ltd.www.tandfonline.com) (c) Different crystal structures in the wet limit can be obtained by packing bubbles into the corner of prism-shaped containers with different opening angles. Shown is the example of an FCC packing in a triangular prism and a BCC packing obtained in a four-sided prism (from [9], reprinted by permission of the publisher Taylor \& Francis Ltd.). (d) Bubbles organise into periodic structures when confined in cylindrical tubes. The type of structure depends on the ratio of tube to bubble diameter [10-12]. (e) Experimental (left) and computational (right) realisation of ordered foam in a tube with rectangular cross-section (reprinted from [13] with permission from Elsevier). (f) "Conformal foams" with elegant bubble arrangements can be obtained by squeezing a mono-layer of equal-volume bubbles into a wedge between two near parallel plates (from [14]). The plate spacing is the smallest at the top of the image, leading to the most strongly squeezed bubbles.

where $<S_{0}>$ is the surface area of the undeformed (spherical) bubbles. Note that various other related non-dimensional quantities are used in the literature.

Since thermal energy is negligible, the macroscopic bubbles are generally trapped in local minima of a very complex energy landscape of the foam. Topological changes, which may lead to a reduction of the global energy via neighbour switching of bubbles, are energetically so costly, that they do not happen spontaneously. Foams may, however, be annealed via mechanical shearing [15,16], by coarsening [17-19] or by the injection of foaming solution (forced drainage [1]), to drive the overall foam structure to a smaller energy. There is thus a strong dependence between the foaming procedure and the resulting bubble packing.

Foam structure may be successfully predicted for the simplest cases of monodisperse periodic foams [2,3] or small bubble clusters [9,20]. However, even though the global packing of more complex structures may not be optimal, the microscopic foam structure, at least in the limit of dry foams, is well defined (Section 2).

Fig. 4 shows the most commonly encountered types of foams. It highlights the roles of liquid fraction $\varphi$ and bubble polydispersity for foam structure. The polydispersity may be defined using the normalised standard deviation of the sphere equivalent bubble radii $R_{i}$, i.e. the radii corresponding to spheres of equal volumes $V_{i}, R_{i}=\left(3 /(4 \pi) V_{i}\right)^{1 / 3}$,

$p_{\sigma}=\frac{\sigma}{\langle R\rangle}=\left[\frac{\left\langle R^{2}\right\rangle}{\langle R\rangle^{2}}-1\right]^{1 / 2}$,

with $\sigma=\left(<R^{2}>-<R>^{2}\right)^{1 / 2}$.

Typically, polydispersities are smaller than $50 \%$ [21] with characteristic distributions being close to Gaussian or Log-Normal. Foams with polydispersity less than $5 \%$ are generally called monodisperse. As will become apparent later (e.g. Section 4), in some cases, rather than using the average radius $\langle R>$, it is more appropriate to use the SauterMean radius $R_{32}$, defined as

$$
R_{32}=\frac{\left\langle R^{3}\right\rangle}{\left\langle R^{2}\right\rangle}
$$




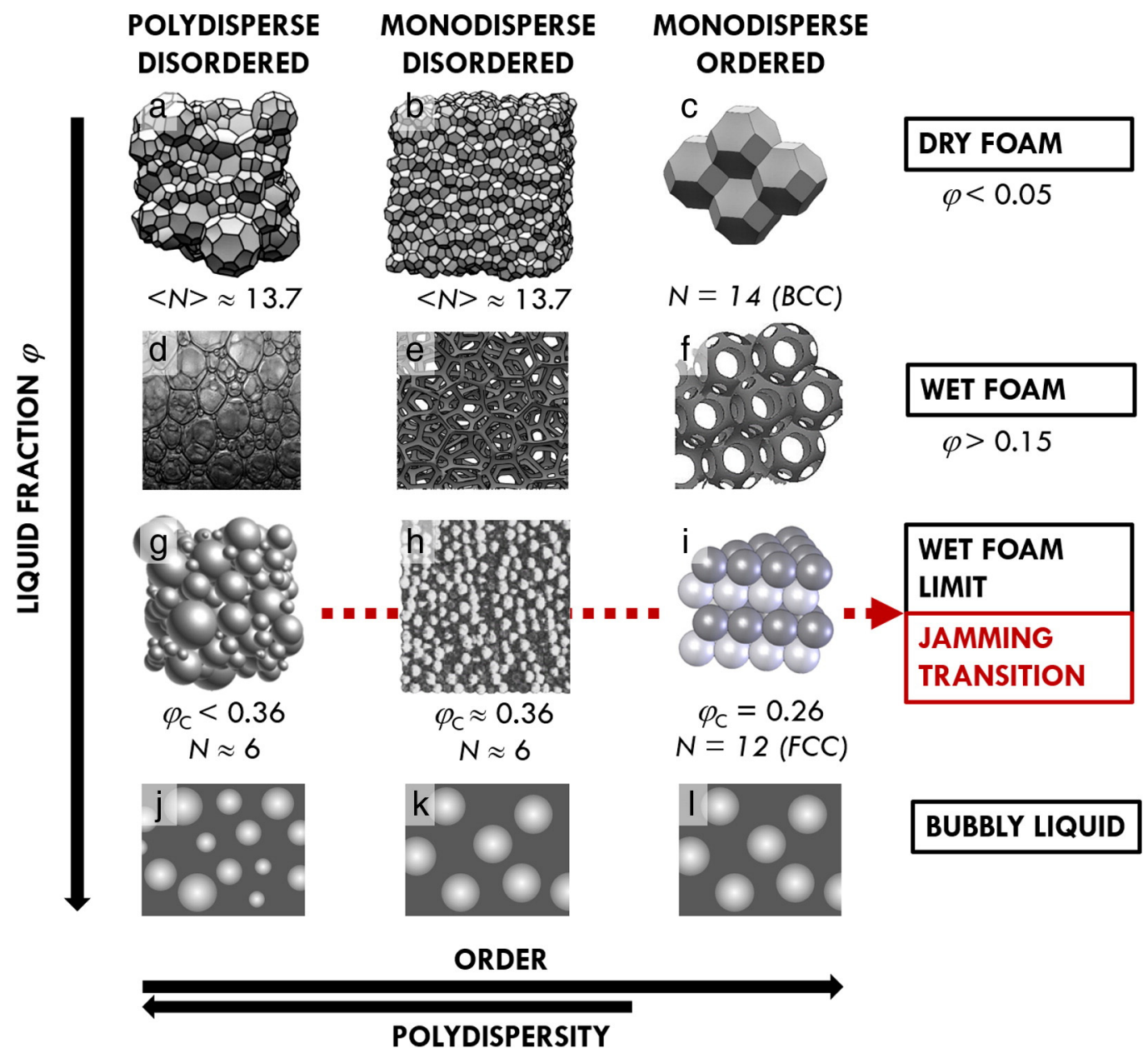

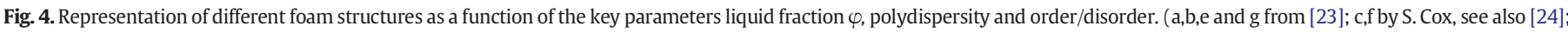
h from [25], i reprinted figure with permission from [26], Copyright 2012 by the American Physical Society). $N$ denotes the number of neighbours of a bubble.

The polydispersity parameter $p_{32}[22]$ is then defined as

$p_{32}=\frac{R_{32}}{\left\langle R^{3}\right\rangle^{1 / 3}}-1=\frac{\langle V\rangle^{2 / 3}}{\left\langle V^{2 / 3}\right\rangle}-1$.

The two polydispersity parameters are linked via $\left(p_{32}+1\right)$ $\left(p_{\sigma}^{2}+1\right)=\left(\frac{\langle V\rangle^{1 / 3}}{\left\langle V^{1 / 3}\right\rangle}\right)^{2}$

In the remaining article we shall look at topology, geometry and energy of different foam structures under the following premises, as justified above

- surface tension $\gamma$ is constant;

- bubbles are considered incompressible;

- foam energy is proportional to surface area.

For the sake of simplicity we shall constrain this description to a small selection of relevant parameters, including the liquid fraction $\varphi$, the scaled energy $\hat{E}$, the relative surface excess $\varepsilon(\varphi)$ and the average number of neighbours of bubbles $\langle N>$. In Section 2 we begin with the analysis of dry foams which contain a small amount of liquid (typically $\varphi<0.05)$ and have therefore bubbles of near polyhedral shapes. In Section 3 we shall discuss foams in the wet limit where they may be approximated as dense packings of soft spheres. In Section 4 we present expressions for $\varepsilon(\varphi)$ for the entire range of liquid fraction. Before concluding in Section 6 we summarise in Section 5 some of the experimental, computational and analytical techniques which are commonly used to investigate the structure of foams.

\section{The dry limit $(\varphi<0.05)$}

The structure of foams with liquid fraction less than about five percent is generally well described by Plateau's laws [1,27,28] (Fig. 5):

1. Three films meet symmetrically under angles of $120^{\circ}$ in channels called Plateau borders.

2. Four such Plateau borders meet symmetrically in junctions (nodes) under the tetrahedral angle of $\operatorname{acos}(-1 / 3) \approx 109.47^{\circ}$ (also called the Maraldi angle).

The liquid in this dry foam limit is mainly contained in the Plateau borders and their junctions (as seen at the top of the foam shown in Fig. 1). For many purposes the film thickness may be considered as infinitesimally thin.

Plateau's rules, which determine foam structure on a local, but not global level, are a consequence of the minimisation of total surface area. They date back to the 19th century Belgian scientist Joseph Antoine Ferdinand Plateau who derived them from observations of soap films trapped in wire frames [27]. In foams beyond the dry foam regime, deviations from Plateau's laws occur, such as stable 8 -fold vertices $[29,66]$ or equilibrium angles between films of less than $120^{\circ}$ [30,31].

The shape of the liquid film separating two bubbles is dictated by the Young-Laplace law

$\Delta P=4 \gamma \kappa$, 


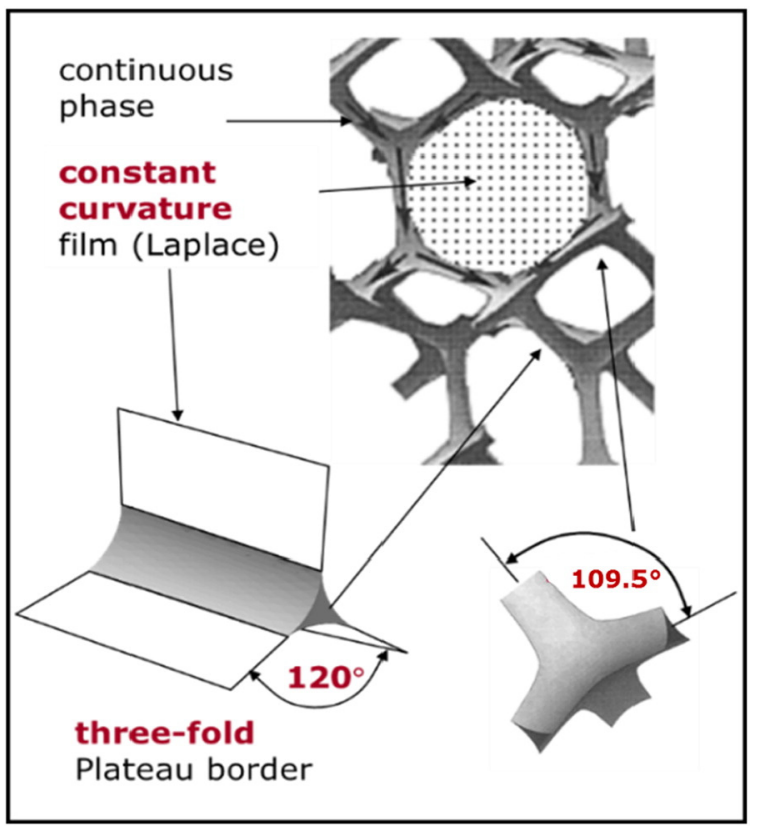

Fig. 5. The topology and geometry of foams in the dry limit $(\varphi<0.05)$ is governed by Plateau's laws and the Young-Laplace law. (The images are results of Surface Evolver simulations [33] and have been kindly provided by S. Cox.).

where $\Delta P$ is the pressure difference between the bubbles. The mean curvature $\kappa$ of the interface is given by $\kappa=\frac{1}{2}\left(\frac{1}{r_{1}}+\frac{1}{r_{2}}\right)$, where $r_{1}$ and $r_{2}$ are the two principal radii of curvature. Since the bubbles within a foam impose a constant volume constraint, pressure differences and film curvatures between neighbouring bubbles adjust in an intricate manner to give the final equilibrium configuration. The result is generally that bubbles in a foam are at different pressures, related to their number of neighbours $N$, which in turn is correlated with their bubble volume, as shown in $[1,28]$. This plays an important role in foam ageing processes driven by gas-diffusion between neighbouring bubbles [32].

The equilibrium rules stated above set important constraints on how bubbles can partition 3D space and also impose numerous statistical constraints on topology and geometry of the bubbles $[1,28]$.

The quest for the structure of monodisperse, periodic foams dates back to 1887, when Lord Kelvin asked the question: how can one divide space into equal-volume cells such that the total interfacial area is minimised $[34,110]$ ? At the time Kelvin and many other scientists tried to construct models of the ether, the hypothesised medium for the propagation of light waves that Einstein finally disposed of. Kelvin thought that the ether might have the structure of a foam. After considering various possibilities his candidate for the optimal structure was the truncated octahedron, as shown in Fig. 6a, whose bubbles have $N=14$ neighbours and are arranged in a BCC structure. In order to adhere to Plateau's rules Kelvin introduced a slight curvature in the 8 hexagons that make up the Kelvin cell, while its 6 square faces are flat.

Unbeaten for over 100 years, it was only in 1994 that Weaire and Phelan [35] managed to compute a structure (Fig. 6b) with $0.3 \%$ less interfacial area. The Weaire-Phelan structure has eight bubbles, of two different types, in a periodic unit, resulting in an average number of $\langle N>=$ 13.5 neighbours. These periodic units fill space when placed at the lattice points of a BCC lattice. All bubbles have equal volume, so this is really a solution to what has been called the Kelvin Problem [34] - although there is no mathematical proof that it is the optimal solution. While several other structures have been computed since which improve upon Kelvin's solution, none of them supersedes the Weaire-Phelan structure [35-37].

Although the Weaire-Phelan structure is energetically favourable $(\hat{E}=5.288)$ over the Kelvin structure $(\hat{E}=5.306)$ (Table 1$)$, it is not easily found or produced in real foams. The first experimental realisation of it
(Fig. 6d) was only achieved 18 years after its theoretical description [8]. A Kelvin foam may, for example, be generated by draining a hexagonally-close packing of spherical bubbles [5,6], which, as discussed in Section 3, forms readily in sufficiently wet foams under gravity. The making of a Weaire-Phelan foam requires the fabrication of a templated container [8] since unlike the Kelvin foam its crystal structure is not compatible with flat container walls. Due to its structural simplicity, the Kelvin structure remains very useful for analytical estimations of different foam properties, a selection of which is provided in Appendix A.

A very crude upper bound on the scaled energy of monodisperse dry foams is given by representing bubbles as cubes $(\hat{E}=6)$, while a lower bound is given by the scaled energy of a simple sphere $(\hat{E}=4.8)$. A more accurate bound is provided by the consideration of a hypothetical bubble (the "ideal bubble" $[39,40]$ ) which has flat faces and the required topology. Such a bubble would have $N=13.4$ and $\hat{E}=5.1$. Even though this bubble is fictitious, it provides a good guide and has inspired the search for an ideal structure of monodisperse foams by pointing towards bubbles with pentagonal faces as good candidates.

The structure of monodisperse foams found in any experiment depends on the details of both set-up, e.g. the boundary conditions (flat or curved container walls, container diameter etc), and procedure (e.g. re-wetting of the foam etc.), thus providing the experimentalist with a certain control over the selection between different structures.

Let us now consider disordered dry foams [1,22,28,36,42]. Fig. 7 to Fig. 9 summarise a few of their properties. Surface Evolver simulations (Section 5.2) of monodisperse disordered foams [36] show that their average number of neighbours $\langle N>$ is approximately 13.7, similar to the value found by Matzke in an experimental study in 1946 [41] (Fig. 7a and Table 1), and also close to the values for the Weaire-Phelan and the Kelvin structure. Increasing polydispersity in such simulations leads to a decrease in both $<N>$ and $\hat{E}$ (Fig. 7b,c). Despite their randomness, such packings feature a number of statistical correlations concerning their local structure $[1,28]$. For example, Fig. 8 , based on simulation data for large bubble clusters, shows that the number of neighbours of a bubble increases with its volume: the bigger a bubble, the more neighbours it has $[28,32,43]$. Also, on average, a large bubble is more likely to be surrounded by smaller bubbles [1,28,44], a correlation related to the Aboav-Weaire law for two-dimensional foams [1].

\section{KELVIN STRUCTURE \\ WEAIRE-PHELAN STRUCTURE}

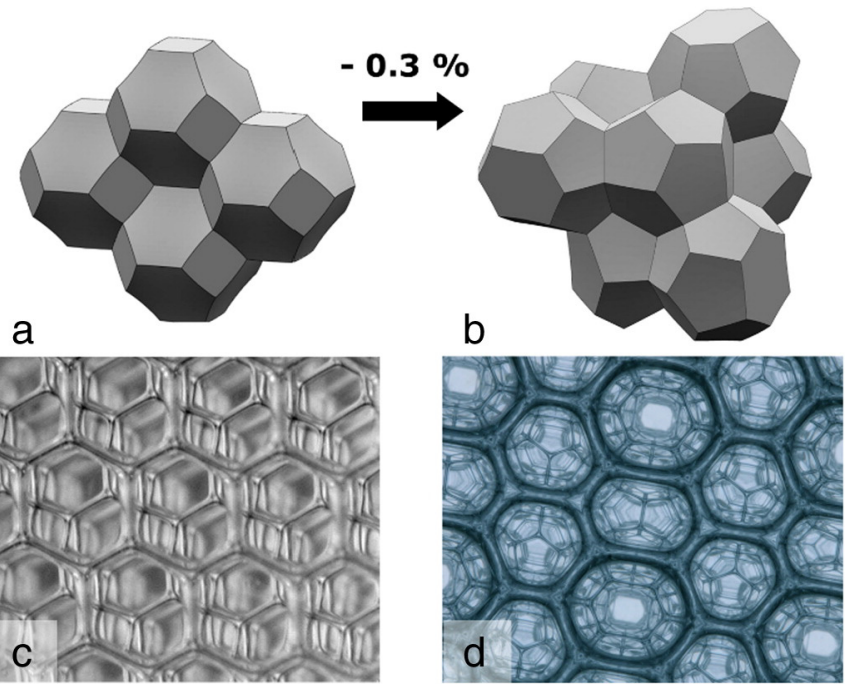

Fig. 6. Surface Evolver simulations by S. Cox $(\mathrm{a}, \mathrm{b})$ and experimental realisations $(\mathrm{c}, \mathrm{d})$ of the Kelvin and the Weaire-Phelan structure. ((c) reprinted from [7] with permission from Elsevier, (d) from [8], reprinted by permission of the publisher Taylor \& Francis Ltd.www.tandfonline.com.) 
Large-scale Surface Evolver simulations of polydisperse random dry foam enable the computation of the ratio $S / V^{2 / 3}$ for every bubble in the packing. As shown on the right of Fig. 9, this reveals that in a foam the bubbles arrange themselves in such a way that their values of $S / \mathrm{V}^{2 / 3}$ are close to a lower bound $\hat{E}^{*}=5.32 \pm 0.04$ [23], independently of the number of neighbours $N[22,45,46]$. This value exceeds those for the monodisperse Kelvin $(\hat{E}=5.306)$ and Weaire-Phelan structure $(\hat{E}=5.288)$. Fig. 9 shows that this is in contrast to the packing of flatfaced Laguerre polyhedra of the same volume distribution where $S / V^{2 / 3}$ depends strongly on $N$. Note that the value of $\hat{E}^{*}$, obtained for polydisperse dry foams, corresponds to $\varepsilon^{*}=\varepsilon(\varphi=0)=\varepsilon^{*}(36 \pi)^{-1 / 3}-1=$ $0.100 \pm 0.008[22,45]$.

Knowing the above, one can combine Eqs. (3) and (7) to give the following explicit relationship between the scaled energy $\hat{E}$ of the foam and its polydispersity $[22,45,46]$,

$\hat{E}=\frac{\hat{E}^{*}}{1+p_{32}}$.

The solid line in Fig. 7c shows that this simple relationship fits well with the results of computer simulations for both poly- and bidisperse foams.

While the vast majority of statistical data on dry foam structure is from Surface Evolver simulations (Section 5.2), it is hoped that desktop X-ray tomography (Section 5.1), recently successfully applied to disordered and ordered wet foams $[17,47-49,4,25]$, can soon also be used for dry foams.

\section{Wet foams at the rigidity loss/jamming transition}

In our discussion of foam under gravity (Section 1) we noted that bubble shapes vary with the local value of liquid fraction, from polyhedral in the dry limit, to spherical in the wet limit, close to the foamliquid interface. Extended columns of wet foam may be obtained by a number of methods, which include the use of bubbles which are much smaller than the capillary length (Section 1), the continuous addition of surfactant solution at the top of a foam ("forced drainage" [1]), or by microgravity experiments [50].

Elastic moduli, yield stress and yield strain decrease with increasing liquid fraction of the foam. They vanish in the wet foam limit at a critical value of liquid fraction $\varphi_{\mathrm{c}}$. The foam then loses its rigidity and might better be described as a dense packing of spherical bubbles. This transition at $\varphi_{C}$ is therefore often referred to as the "rigidity loss transition" upon increasing the liquid fraction, or, equivalently as the "jamming transition" upon decreasing liquid fraction around $\varphi_{\mathrm{c}}$.

The critical value of $\varphi_{C} \approx 0.36$ for random monodisperse foams is familiar to researchers of granular materials, where the Bernal packing fraction is $0.64=1-0.36$. It corresponds to the packing density at which a random collection of equal-volume hard spheres jam together [51-54] (Fig. 4). In polydisperse foams the critical liquid fraction decreases only slightly from the value of 0.36 for the modest polydispersities which are typically obtained from many experimental foaming techniques $[21,55]$.

Table 1

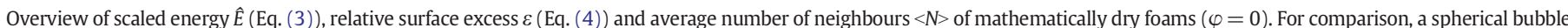
has $\hat{E}=4.836$

\begin{tabular}{|c|c|c|c|c|}
\hline Type of structure & Scaled energy $\hat{E}$ & Relative surface excess $\varepsilon(\varphi=0)$ & Number of neighbours $\mathrm{N}($ or $<N>$ ) & Year + Ref \\
\hline Cubic tiling & 6 & 0.241 & 6 & \\
\hline Ideal bubble (not space filling) & 5.1 & 0.055 & 13.4 & $1992[39,40]$ \\
\hline Kelvin & 5.306 & 0.097 & 14 & $1887[34]$ \\
\hline Weaire-Phelan & 5.288 & 0.093 & 13.5 & $1994[35]$ \\
\hline Random monodisperse foam & $5.330 \pm 0.006$ & $0.102 \pm 0.001$ & 13.7 & $1946 / 2003[36,41]$ \\
\hline Random polydisperse foam (for $p_{32}<0.5$ ) & $3.6<\hat{E}<5.33$ & $0.100 \pm 0.008$ & $11.4<<N><13.7$ & $2004[22]$ \\
\hline
\end{tabular}
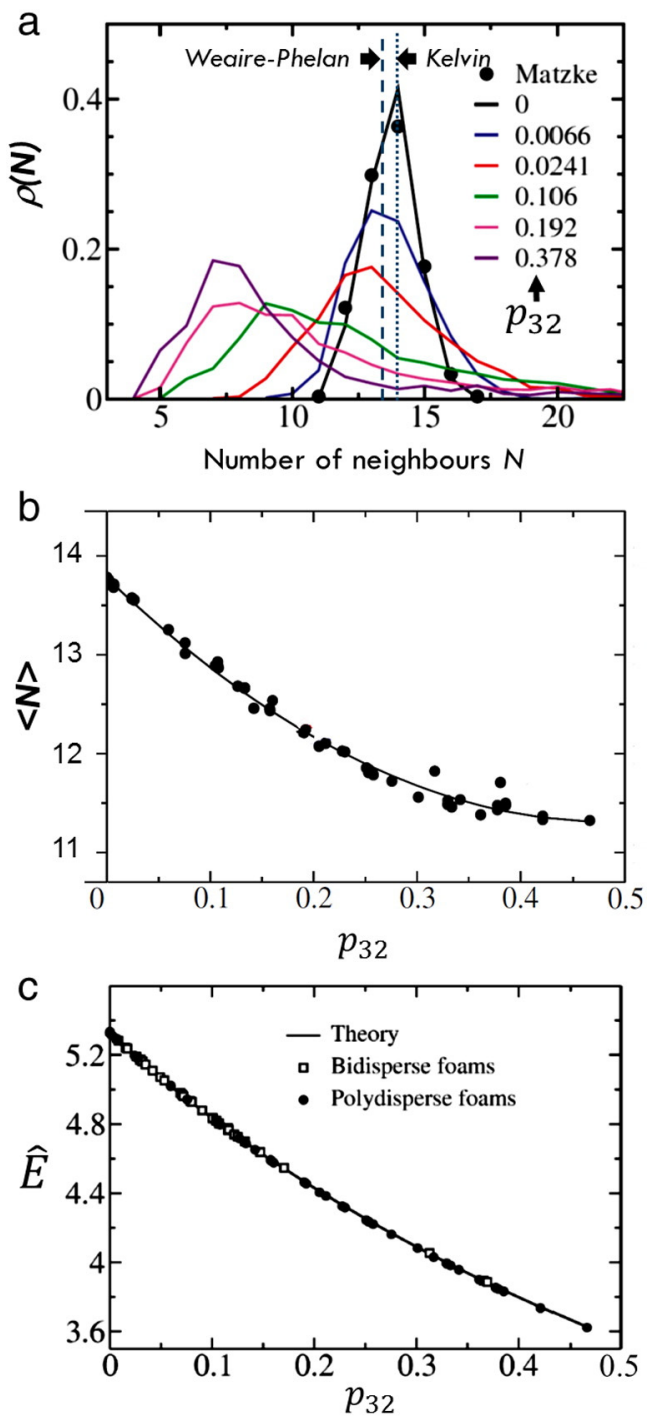

Fig. 7. (a) Probability distribution of the number of bubbles with $N$ neighbours in a foam with different polydispersities $p_{32}$, defined in Eq.(7) (reprinted figure with permission from [22] Copyright 2004 by the American Physical Society). Also shown is the only experimental data available for monodisperse disordered foams, taken by Matzke [41]. (b) Average number of neighbours $<N>$ as a function of polydispersity $p_{32}$ (adapted from [23]). (c) Variation of scaled energy $\hat{E}$ with polydispersity $p_{32}$ for polydisperse and bidisperse foams. The solid line is given by Eq. (9). (From [22,23]).

At the jamming transition, which is also called the isostatic point [52], bubbles begin to contact their nearest neighbours (excluding any "rattlers", i.e. small bubbles that might sit loosely in the crevices between densely packed larger bubbles). It is reasonable to associate an average number of $\langle N\rangle=6$ nearest neighbours of a bubble by analogy to sphere packings, for which various numerical and experimental studies exist $[52,53,56]$. This has been shown to hold in the case of non-Brownian emulsions $[57,58]$. However, unlike in the case of two-dimensional foams where $<N>=4$ at jamming [59], there is as yet no corresponding 


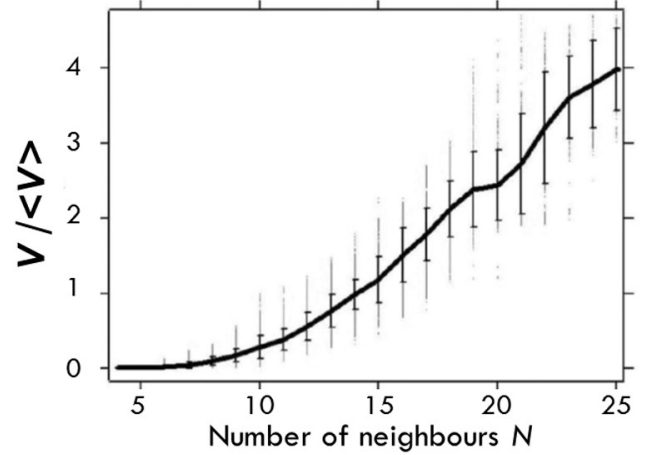

Fig. 8. Normalised bubble volume as a function of the number of neighbours $N$ in a polydisperse foam, showing that both are strongly correlated (reprinted from [32] with permission from Elsevier).

experimental or numerical 3D data for foams. Also, while for frictionless spheres constraint counting arguments may be used to arrive at an average of $\langle N\rangle=6$ nearest neighbours, it has been shown that the extension of such arguments to (even infinitesimally) non-spherical objects may be non-trivial [60].

A striking observation is the spontaneous crystallisation of equalsize bubbles of a few hundred microns in diameter into a wet ordered foam, an example of which is shown in the photograph on the left of Fig. 10. This phenomenon seems quite independent of the specific foam production technique [5]. Although originally reported in the 1940s by Bragg and Nye [61], the observation was not pursued by others until recently and is still not fully understood. While crystallisation is observed close to a boundary or liquid interface as soon as the foam is made [4-7], recent data also shows the growth of crystalline regions in the bulk of a monodisperse foam over the course of a few days. The crystalline bubble packings are of the types $\mathrm{ABC}$ and $\mathrm{ABA}$, as shown on the right of Fig. 10, corresponding to face centred cubic (FCC) and hexagonally close packed (HCP) structures $[5-7,61]$ in which each bubble is in contact with $N=12$ nearest neighbours. FCC and HCP layering can also combine to form random close packed (RCP) structures which surprisingly have not yet been observed for wet foams.

The choice of the close-packed structure over other types of packings is not surprising. In the wet limit the buoyancy force acting on a bubble is negligible in comparison to the surface tension forces which resist bubble deformation. The bubbles can therefore be approximated as hard spheres, and the minimisation of the potential energy of the bubbles due to buoyancy will create a packing of minimal density [62].

Although all crystalline closed-packed structures have the same packing density, a number of different experiments showed a clear preference of ABC (FCC) arrangements [4,6,25]. Such a preference of
FCC over alternative closed-packed structures is known from other fields, when entropic contributions play a role (in the presence of Brownian motion, for example) or when interaction energies extend beyond nearest neighbour interactions. However, none of these influences are expected to play a role in the type of bubble packings considered here.

Computer simulations for buoyancy-driven soft spheres aggregating underneath a flat surface, based on the solution of the Navier-Stokes Equation using an immersed boundary method [63], showed a similar preference of FCC. This has been attributed to a reduced mechanical stability of non-FCC structures: forces between neighbouring spheres are not transmitted in the same way in FCC and HCP packings, making HCP packings noticeably less stable [26].

Both soft sphere simulations [64] (Fig. 11a) and experiments (Fig. 11b,c) [4] have shown that the gravity-driven ordering of spheres may be rapidly lost away from the crystallising influence of a boundary. As the sample thickness grows over the course of the experiment due to the continued agglomeration of bubbles, ordered arrangements give way to random Bernal packing with $\varphi_{\mathrm{C}} \approx 0.36[4,64]$. This may be due to the fact that the system is jammed more rapidly by the newly arriving bubbles than it has time to re-organise the bubbles into an energetically more favourable, ordered configuration. However, it has been shown in experiments using X-ray tomography (Section 5.1) that over the course of a few days, these disordered samples may crystallise [25], as shown in Fig. 11d.

Many questions remain regarding the observed spontaneous ordering in monodisperse wet foams and the above-mentioned slow recrystallisation. How much coarsening or drainage is sufficient for the restructuring of a sample? Do slight mechanical disturbances of the sample anneal the foam to a more optimal configuration? Does one need to take into account more realistic bubble interaction potentials, as discussed in Section 4? All points to a clear need for more experiments and for more realistic simulations.

\section{Interpolating between the limits of wet and dry foams}

\subsection{Foam structure and bubble interaction}

While structure and properties of foams are fairly well understood in both the dry and the wet limit (Sections 2 and 3), many questions remain how to interpolate between these extremes of liquid fraction.

Starting from the dry limit and increasing the liquid fraction one finds experimentally, and from Surface Evolver simulations [29], that Plateau's laws (Section 2) can be violated and that 8-fold vertices become stable. The critical value of liquid fraction at which this occurs appears to depend on the experimental details. For soap films attached to a cubic wire frame a value of $\varphi \approx 0.02$ has been found [65]. In ordered bulk foam a transition from predominantly Kelvin (BCC) structure

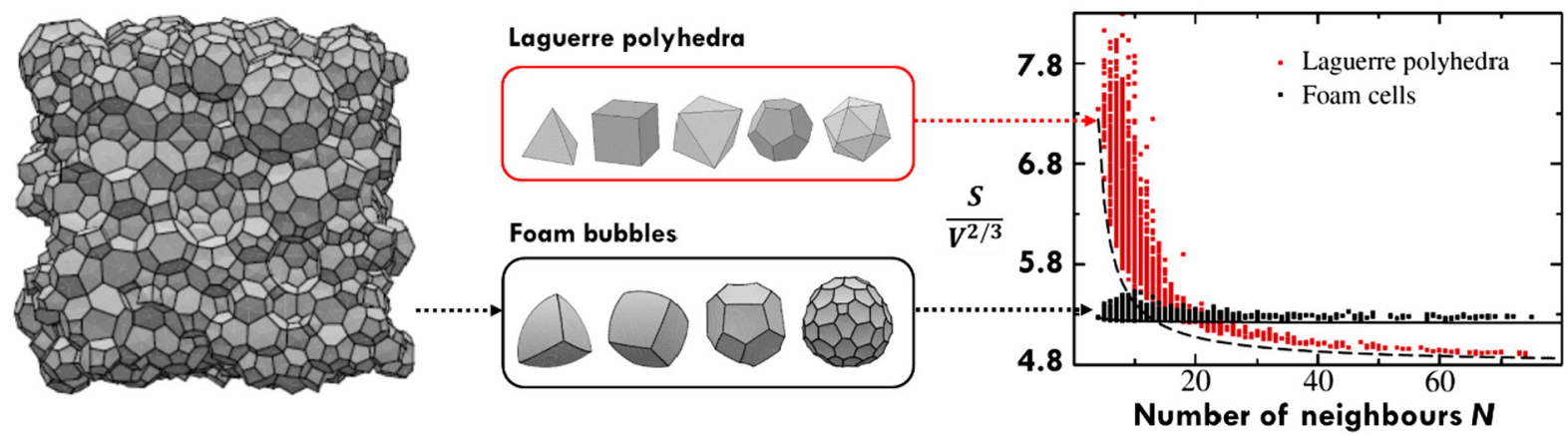

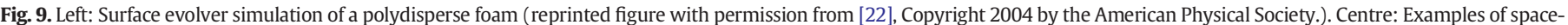

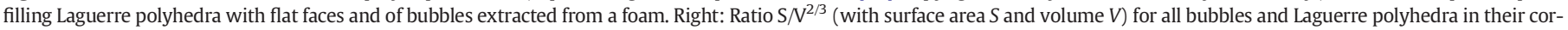

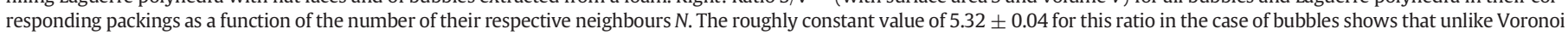
cells, bubbles adjust their shape when packed together. (Adapted from [23]). 


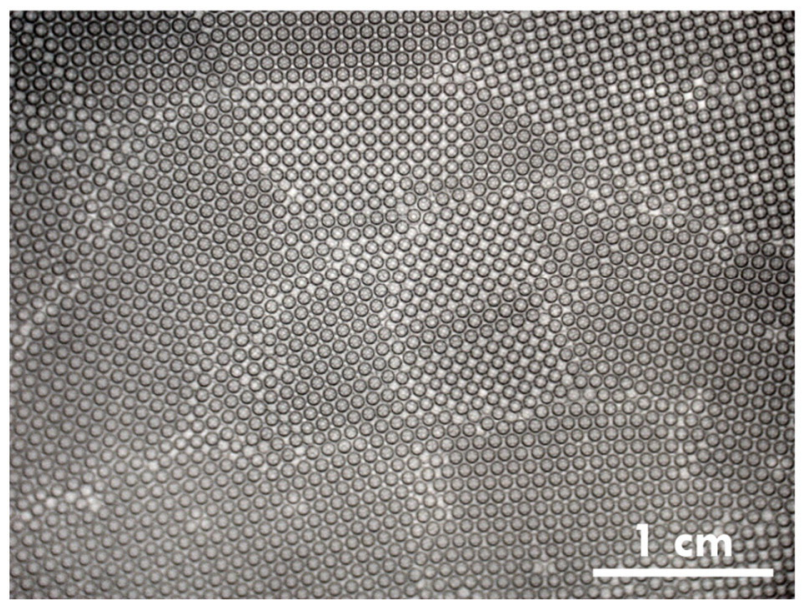

FCC

(Face Centered Cubic)

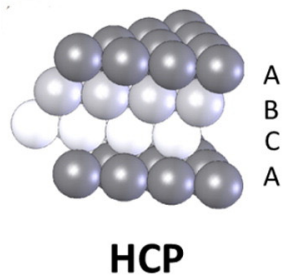

(Hexagonally Close-Packed)

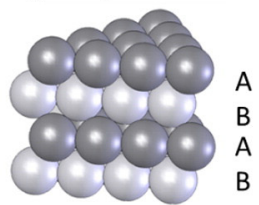

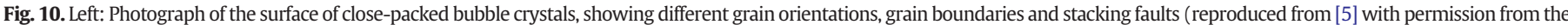
Royal Society of Chemistry). Right: Arrangement of spheres in FCC and HCP packings (reprinted figure with permission from [26], Copyright 2012 by the American Physical Society).

(with four-fold vertices) to predominantly FCC structure (with eightfold vertices) has been observed in the range $\varphi=0.06 \pm 0.02$ [6], where both Kelvin and FCC structures are observed to coexist. These values are in good agreement with Surface Evolver simulations which predict a crossover of the surface excess $\varepsilon$ at $\varphi=0.063$, as shown in Fig. 12. Note that this is below the value of $\varphi \approx 0.11$ which marks the loss of the four-sided face in a Kelvin foam [66]. Upon increase of the liquid fraction one also observes deviations from angles prescribed by Plateau's laws (Section 2) at Plateau borders and vertices due the increasing effective line tension of the liquid-carrying channels [30,31]. For a review of a number of instabilities in liquid foam see [67].

When decreasing the liquid fraction away from the wet limit, foams are often regarded as frictionless, granular matter since the bubbles are sufficiently large so that Brownian motion can be neglected (Section 1). Furthermore, the absence of static friction makes the jamming transition in foams very well defined in contrast to other granular systems [51-53]. Since bubbles are deformable they are often modelled as soft spheres, with a pairwise interaction potential $U(\xi)$

$U(\xi) \sim F(\alpha) \xi^{\alpha}$

where $\xi$ is the normalised overlap of the spheres and $\alpha$ controls the type of potential $[51,52,57,68-71]$. For example, $\alpha=2$ for a harmonic potential and $\alpha=5 / 2$ for a Hertzian potential. Using such kinds of pairwise interaction potentials one can show that the change of the number of neighbours, $\Delta N$, scales with distance $\Delta \varphi$ to the jamming point as a power law $[51,52,56]$

$\Delta N \sim \Delta \varphi^{1 / 2}$

independently of the value of $\alpha$ [51], with $\langle N>\approx 6$ at the jamming point (see Fig. 13). This has been confirmed experimentally for the case of nonBrownian emulsions $[57,58]$. No equivalent data is yet available for threedimensional foams. Experimental data for two-dimensional foams (a monolayer of foam between a glass plate and a liquid surface) shows a similar power law scaling, but the value of the exponent depends on how one defines a two-dimensional liquid fraction [59].

Ultimately such a scaling in contact numbers above the jamming point is also responsible for the power law behaviour of mechanical quantities, such as shear moduli or osmotic pressure in foams (and emulsions) [57,69,72-74].

However, since bubble interaction in foams is controlled by the overall change of surface area upon compression, the detailed form of the potential is more complicated than suggested by Eq. (10) [70,75]. For example, in the case of periodic foam structures with $N$ neighbouring bubbles analytical calculations using the Z-cone model $[75,76]$ show that the relative surface excess is given by

$$
\varepsilon(\varphi) \sim-\frac{N}{18\left(1-\varphi_{c}\right)^{2}} \frac{\left(\varphi_{c}-\varphi\right)^{2}}{\ln \left(\varphi_{c}-\varphi\right)}
$$

This expression agrees very well with data obtained from corresponding Surface Evolver simulation for small deformations [70,75] (see Fig. 16 later). Eq. (12) shows that bubble interaction is nonpairwise, i.e. it depends on the number of neighbours of a bubble. Moreover, even though it contains a harmonic term, it is "logarithmically soft" for small deformations $[70,75]$. Approximations of the type of potential corresponding to Eq. (12) have been made in the past by using the form of Eq. (10) with exponent $\alpha$ depending on the coordination number $N$ of the bubbles $[68,77]$.

\subsection{Surface energy and osmotic pressure}

The relative surface excess $\varepsilon(\varphi)$ over the entire range of liquid fraction can be obtained from Surface Evolver simulations, but for the moment computations are restricted to ordered foams, since the structural instabilities discussed at the outset of this section are difficult to handle computationally. $\varepsilon(\varphi)$ cannot be measured directly in experiments, but may be derived from measurements of the so-called osmotic pressure of a foam, or from the liquid fraction profile of a foam under gravity, as we will show below and in Appendix B.

The concept of an osmotic pressure $\Pi(\varphi)$ for foams and emulsions is due to the pioneering work of Henry Princen [78-80] who also provided the first measurements and some semi-empirical relationships. One of the major advantages of this quantity is that it is directly accessible to experimental measurement. Note that unlike its counterpart in physical chemistry, the osmotic pressure for foams is not driven by entropy, but by the energetic contributions of the interfaces.

In the case where gas and liquid are treated as incompressible, $\Pi$ is given by

$\Pi=-\gamma\left(\frac{\partial \mathrm{S}}{\partial V_{f}}\right)_{V_{g a s}=\text { const. }}$,

where $S$ is the total surface area of the bubbles within a foam volume $V_{f}$, and $V_{\text {gas }}$ is the gas volume, which remains fixed. As sketched in Fig. 14, the osmotic pressure is the force per unit area on a semi-permeable membrane which presses on the foam, letting liquid pass, but not the bubbles. The resulting pressure on the membrane is due to the fact 
a
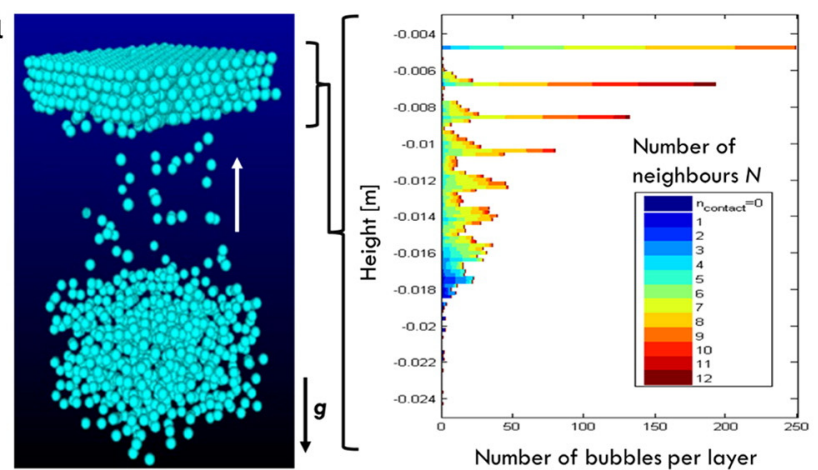

Number of bubbles per layer

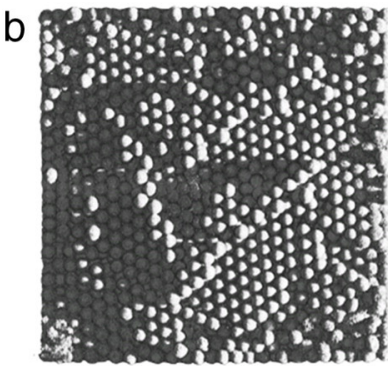

Bottom thin sample $(<15$ layers)

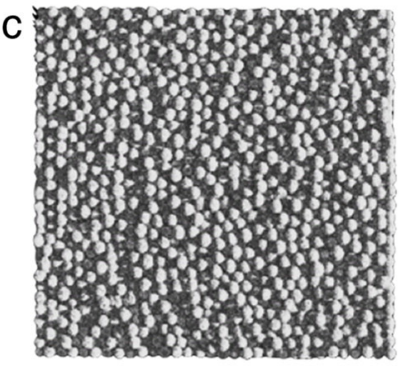

Bottom thick sample (> 15 layers)
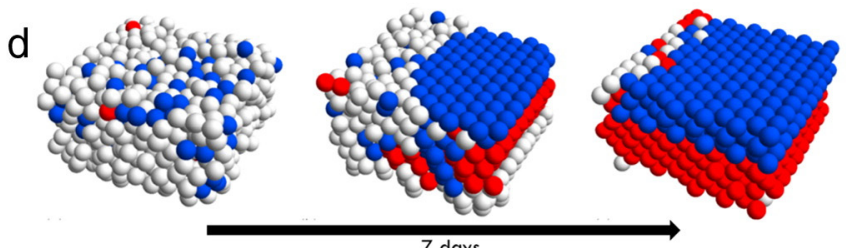

Fig. 11. Crystallisation in soft sphere simulations (a) and in experiments with equal-volume bubbles (b-d). (a) Computer simulations using soft spheres and an immersed boundary method (unpublished data from [64]) show that ordering generally only occurs over a small number of layers close to an interface. This is similar to the situation in experiments with monodisperse bubbles of a few hundred microns in diameter. X-ray tomography reveals ordering close to the surface (b) and disorder (Bernal packing) sufficiently far away from it (c). In addition to such spontaneous crystallisation, monodisperse foams may also crystallise over the course of several days, and in bulk regions, as shown in the X-ray data displayed in (d) (reproduced from [25] with permission from the Royal Society of Chemistry).

that removing liquid from a foam deforms the bubbles, increasing their surface area $S$ and therefore the surface energy stored in the foam. If the foam contains sufficient liquid for the bubbles to lose contact, $\partial S / \partial V_{f}=$ 0 , i.e. $\Pi=0$. Hence, a second major advantage of the osmotic pressure is that it can be used to unambiguously define the jamming transition as the point where $\Pi$ becomes non-zero.

$$
\text { Using } \frac{\partial}{\partial V_{f}}=\frac{V_{g}}{V_{f}^{2}} \frac{\partial}{\partial \varphi} \text {, with } V_{f}=\frac{V_{g}}{1-\varphi} \text { and } \frac{V_{g}}{S_{c}}=\frac{\left\langle R^{3}\right\rangle}{3\left\langle R^{2}\right\rangle}=\frac{R_{32}}{3} \text {, Eq. (13) can be }
$$
re-expressed as

$\Pi(\varphi)=-\frac{\gamma}{R_{32}} 3(1-\varphi)^{2} \frac{\mathrm{d}}{\mathrm{d} \varphi}\left(\frac{S(\varphi)}{\mathrm{S}_{0}}\right)=-\frac{\gamma}{R_{32}} 3(1-\varphi)^{2} \frac{\mathrm{d}}{\mathrm{d} \varphi}(\varepsilon(\varphi))$,

where $S_{0}$ is the total surface area of the undeformed (spherical) bubbles and $R_{32}$ is the Sauter mean radius (Eq.(6)). This equation thus relates osmotic pressure to the change in relative surface excess $\varepsilon(\varphi)$. It also shows that the prefactor $\gamma / R_{32}$ sets a natural pressure scale, which is why in the following the osmotic pressure is presented in its nondimensionalised form

$\hat{\Pi}=\frac{R_{32}}{\gamma} \Pi$

The relative excess energy of the foam may then be obtained by integration of Eq. (14),

$\varepsilon(\varphi)=\int_{\varphi}^{\varphi_{c}} \frac{\hat{\Pi}\left(\varphi^{\prime}\right)}{3\left(1-\varphi^{\prime}\right)^{2}} d \varphi^{\prime}$

The variation of osmotic pressure with liquid fraction can be obtained experimentally, either from direct measurements using osmometers (see Höhler [6] and Mason [68]), or via the measurement of liquid fraction profiles of foams in equilibrium under gravity $[77,79]$, as detailed in Appendix B. Fig. 15 displays such data for $\tilde{\Pi}(\varphi)$, on a double-logarithmic scale.

Two distinct curves are visible in Fig. 15 which correspond to ordered monodisperse foam, for which the osmotic pressure vanishes at a critical liquid fraction $\varphi_{\mathrm{c}}=0.26$, and to disordered polydisperse foams for which $\varphi_{\mathrm{c}} \approx 0.36$ (Section 3).

For reliable integration of the data of Fig. 15 in order to obtain $\varepsilon(\varphi)$ via Eq. (16) it would be desirable to have an analytic description of $\tilde{\Pi}(\varphi)$ at hand. In the dry limit such a description is straightforward. A change in liquid fraction simply leads to a change in the crosssectional area of the Plateau borders (Section 2), independently of the foam structure, and therefore to $\mathrm{d} S / \mathrm{d} \varphi \sim \varphi^{-1 / 2}$. Since in this limit one can approximate $(1-\varphi) \approx 1$, one finds from Eq. (14) that

$\tilde{\Pi} \sim \varphi^{-1 / 2}$

The success of this approximation can be seen clearly in Fig. 15 where all data follow a power law with slope $-1 / 2$ in the dry limit.

Approximations for the osmotic pressure in the wet limit may be obtained using models of the interaction potentials, such as the ones discussed at the beginning of this section $[6,69,75,77]$.

Empirical forms for the variation of osmotic pressure with liquid fraction may then be constructed by simple multiplication of relevant expressions for the dry and the wet limit [77]. For most practical purposes, the following simple relationship, proposed by Höhler at al. [6] for the case of monodisperse, ordered foams,

$\tilde{\Pi}(\varphi)=K \frac{\left(\varphi_{c}-\varphi\right)^{2}}{\varphi^{1 / 2}}$

is sufficient, also for disordered foams [77] (see solid lines in Fig. 15). Even though it is a fairly crude approximation of the wet limit, it has the merit of being a simple expression in comparison to more realistic expressions [77]. The prefactor $K$ is obtained via application of the socalled "Princen criterion" $[77,80]$, which is given by integration of Eq. (14) over the entire range of liquid fraction

$\int_{0}^{\varphi_{c}} \frac{\hat{\Pi}}{3(1-\varphi)^{2}} d \varphi=-\frac{1}{S_{0}} \int_{S(\varphi=0)}^{S_{0}} d S=\left[\frac{S(\varphi=0)-S_{0}}{S_{0}}\right]=\varepsilon(0)=0.100 \pm 0.008$,

where the value of $\varepsilon(\varphi=0)$ has been discussed in Section 2 (Table 1). Numerical integration using Eq. (18) in Eq. (19) leads to $K=7.3$ for monodisperse, ordered foams ( since $\varphi_{\mathrm{c}}=0.26$ ), while for disordered foams one finds $K \approx 3.2$ since $\varphi_{\mathrm{c}} \approx 0.36$.

With pre-factor K established, the variation of $\varepsilon(\varphi)$ with liquid fraction based on experimental data for different types of foams can then be computed from Eq. (16). Fig. 16 shows this, together with data from Surface Evolver simulations and results from the Z-cone model for $N=12$ (FCC), which is only a good approximation of numerical data in the wet limit. Experimental data for both ordered and disordered foams, which is well described by Höhler's empirical relationship Eq. (18), is indicated by the broad grey lines to highlight its semiempirical nature. A similarly good agreement can be obtained based on the liquid fraction profile as given in Eq. (29) (Appendix B). Note that the relative surface excess for ordered foam lies below the values 


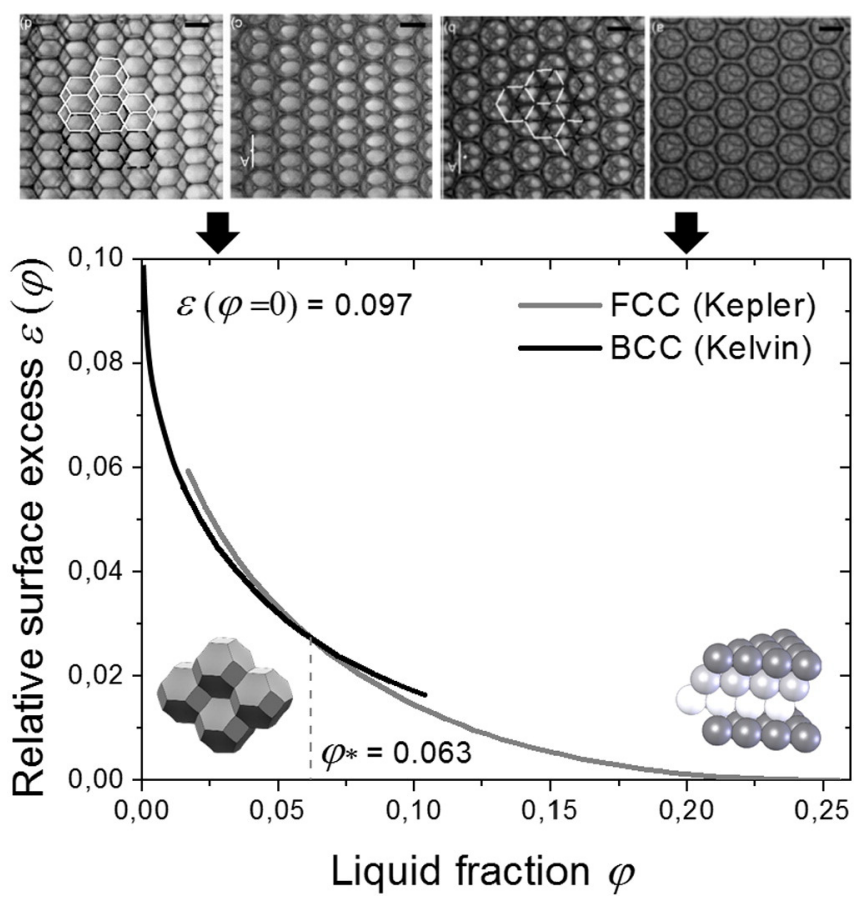

Fig. 12. The transition between ordered dry (BCC) and wet (FCC) foam. Top: Sequence of photographs of experimental samples (Reprinted with permission from [6] Copyright 2008, American Chemical Society.). Bottom: Results of Surface Evolver computations for the relative surface excess $\varepsilon(\varphi)$ (Eq. (4)) of the foam as a function of liquid fraction $\varphi$ showing a cross-over between the FCC and BCC structure at $\varphi \approx 0.063$.

of Surface Evolver calculations and Z-cone model for FCC. This deviation is significantly reduced when using more realistic (yet more complex) models for the osmotic pressure in the wet limit [77]. No Surface Evolver data is yet available for disordered wet foams.

\section{Techniques for foam structure characterisation}

\subsection{Experimental techniques}

Foam structure depends on the local liquid fraction $\varphi$, which may be determined using a variety of experimental techniques. For a column of foam floating on a liquid pool of foaming solution the average liquid fraction of the foam can be determined using Archimedes' principle

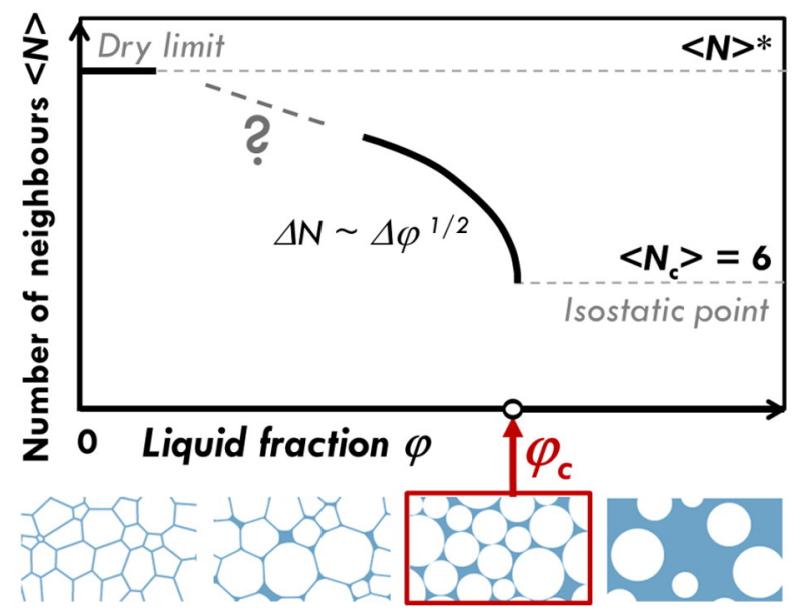

Fig. 13. Suggested variation of average number of neighbours $<N>$ with liquid fraction. While $\left\langle N_{\mathrm{c}}>\approx 6\right.$ at the jamming point, simulations and experiments suggest a power law scaling for decreasing liquid fraction. In the dry limit, the value for $<N\rangle^{*}$ depends on the structure of the foam (Section 2).
[1] by measuring the depth of the immersion of the column. An elegant way to perform the measurement employs the use of a U-tube filled with foam and foaming solution. The amount of liquid on both sides is then equal.

An estimate of the local liquid fraction may be obtained by visual inspection and photography of the Plateau borders in contact with the container wall holding the foam. This approach works best for dry foams thanks to their well-defined geometry (Section 2). Various techniques have been used to determine the size of the surface Plateau borders, the most precise being the one proposed by Garrett et al. [108, 109]. It consists of imaging the foam surface with a lens of large focal length through a $90^{\circ}$ prism, glued to the outside of the container wall. Light reflected by the curved interfaces of the Plateau borders does not enter the camera; the surface Plateau border network therefore appears in black.

The most widely used technique for determining liquid fraction is that of measuring the electrical conductivity of the foam. In the dry limit, there is a linear relationship, $\sigma=\varphi / 3$, first derived by Lemlich [1]. Here, $\sigma$ is the relative conductivity, i.e. the conductivity of the foam, divided by the conductivity of the bulk liquid. Non-linear corrections are required for increasing liquid fraction, and Feitosa et al. [81] have established reliable semi-empirical relationships for both $\sigma(\varphi)$ and $\varphi(\sigma)$.

$\mathrm{X}$-ray (or $\gamma$-ray) radioscopy is a more advanced technique that results in two-dimensional profiles of liquid fraction. It is based on the attenuation of X-rays by the liquid phase and has been employed for both aqueous and liquid metal foams $[82,83]$. Since the X-rays are not scattered by the foamed liquid, the difference in emitted and detected intensity is directly related to the total amount of liquid which the Xrays have traversed.

Information about bubble sizes in a foam may be obtained in a number of ways. Photography of foam in contact with a flat transparent interface (glass, perspex) can be used to obtain the size distribution of surface bubbles. Inferring the bulk distributions from this is not straightforward since bubbles organise differently at the flat container wall than in the bulk foam $[84,85]$ - but often it gives a good approximation of the average bubble size. Bubble size distributions in bulk foams may be obtained by placing extracted foam samples on a liquid surface or between two parallel glass plates, with spacing less than the average bubble diameter [86]. Image analysis of the resulting single bubble layer or quasi-2D foam leads to a volume distribution of bulk bubbles. A non-

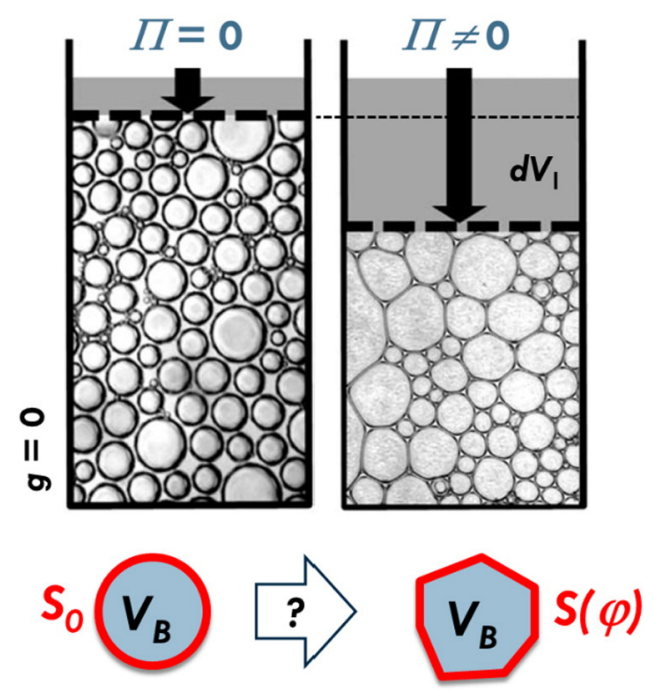

Fig. 14. Princen's Gedankenexperiment for the definition of an osmotic pressure $\Pi$ for foam. The foam is kept in a container with a movable membrane which is permeable to liquid, but not to bubbles. Extraction of liquid from the foam requires bubble deformation. Since this is associated with an increase of interfacial area, a finite osmotic pressure needs to be applied to the membrane. 


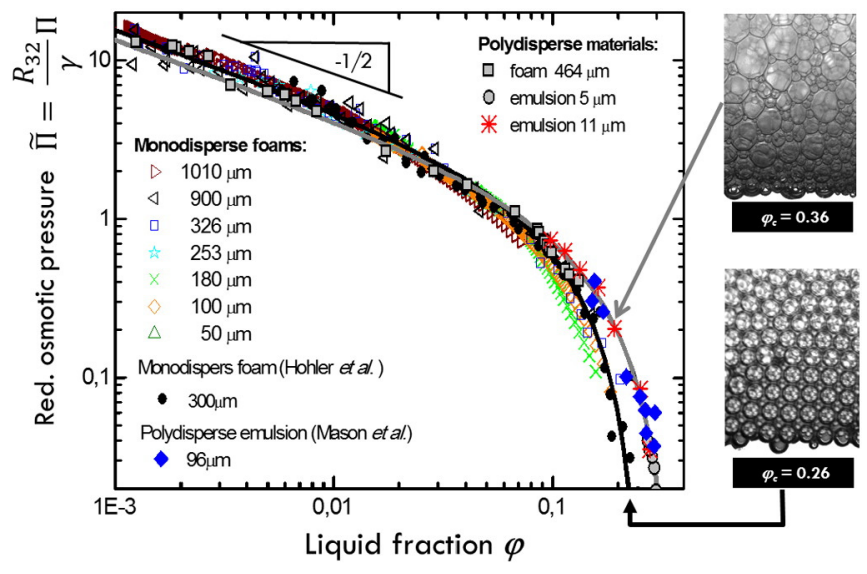

Fig. 15. Reduced osmotic pressure as a function of liquid fraction obtained from integration of the data of Fig. 20 in Appendix B using Eq. (26). Data from Höhler et al. [6] and Mason et al. [68] have been added. These were obtained for foams and emulsions using osmometers. The legend gives the Sauter-Mean radii $R_{32}$ of the bubbles/droplets used. The solid lines correspond to Eq. (18) (with $K=7.3$ and $\varphi_{\mathrm{c}}=0.26$ for the monodisperse case $\left\{\right.$ black line \} and $K=3.2$ and $\varphi_{\mathrm{c}}=0.36$ for the disordered case grey line ).

invasive probing of bulk foam may be obtained via light scattering experiments [87]. The smaller the bubbles of a foam, the stronger light is scattered (demonstrated by the whiteness of shaving foam). From knowledge of the intensity of light which is lost via scattering, together with the determination of the liquid fraction using one of the techniques above, one can obtain the average bubble size [87].

Obtaining information on the details of foam structure is harder. Classic photography has been used extensively under numerous lighting and imaging conditions but is successful only for imaging a few bubble layers. Access to bulk structure is hampered by the diffusive nature of light propagation through a foam. The multiple reflections and refractions that light undergoes often lead to complex image features [88] which can be hard to interpret. So while photography is appropriate for a general visual impression (see for example Fig. 1) it generally fails for quantitative and potentially automated analysis of foam structure.

While confocal microscopy can be used to obtain high-resolution information about the structure of index-matched emulsions [89], this is not possible for foams due to the strong mismatch of the refractive index between the gas and the liquid. The most significant progress in the visualisation and characterisation of 3D foams has been made with the development of tomographic techniques. Initial work using optical [90-92] or MRI tomography [93] is now being followed up by X-ray tomography. This allows for the determination of detailed bulk foam structure and lead, for example, to the identification of crystallinity within a sample, as shown in Fig. 11d [25]. The ever increasing resolution and sensitivity of X-ray detectors recently also enabled the acquisition of 3D images of dry aqueous foams, as shown in Fig. 17 [4,47,49]. While films are still too thin to be detected, one obtains a full presentation of the Plateau border network which is then processed numerically in order to reconstruct the full foam structure for analysis [4,17,47-49].

\subsection{Simulations}

Computer simulations have played a vital role in advancing our understanding of foam structure by providing reliable, quantitative information well before the access to detailed experimental data for 3D foam structure.

Although the principle ultimately responsible for the structure of foams in equilibrium is readily stated, namely the minimisation of surface energy for given constraints (bubble volume distribution, liquid fraction, boundary conditions for confined foams, gravity, etc.), the numerical realisation is a formidable task. The most successful tool which has been used for this purpose is the Surface Evolver software [33] which has been

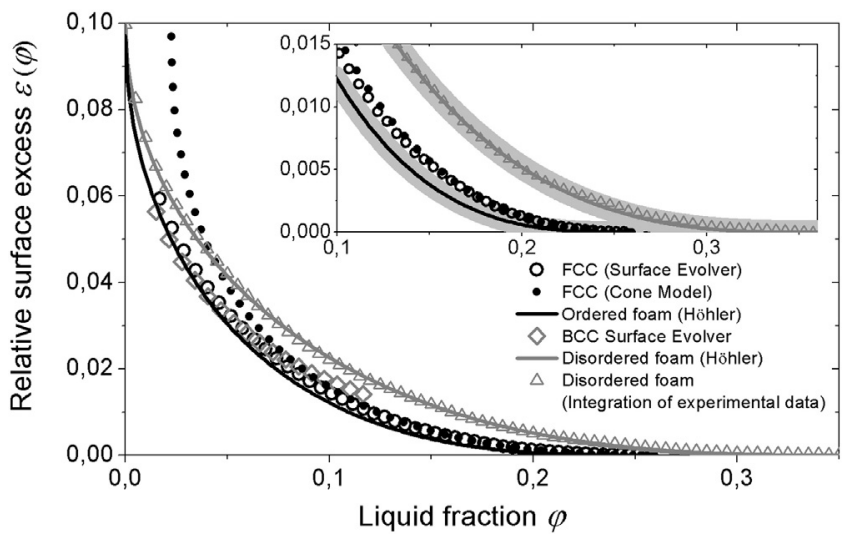

Fig. 16. Overview of the variation of the relative surface excess $\varepsilon(\varphi)$ with liquid fraction for different foam structures obtained from different models, experiments and Surface Evolver Simulations.

provided to the foam community by Ken Brakke for more than 20 years in the most altruistic manner. The curved foam films and foam-liquid interfaces are represented by tessellations of small planar triangles. The minimisation of total surface area is then achieved via an iterative process using a gradient descent algorithm. We have referred to such simulations, and shown examples, throughout this article.

The simulation of wet foams using the Surface Evolver remains challenging. This is particularly so for disordered samples, which require manually dealing with individual topological changes as liquid fraction is varied. Investigations close to the jamming transition commonly simulate the foam as a dense packing of spheres which interact via a pairwise potential [26,51-53,71].

Alternative computational approaches include the Potts Model, which is a Monte Carlo technique [19,94,95]. This enables the gathering of statistical information on sample sizes that are out of reach for Surface Evolver simulations. However, this rule-based model with its probabilistic approach might be criticised for its level of abstractness.

A promising new model was recently developed by Sethian and Saye [96] and applied to the temporal evolution of a bubble cluster. This includes the thinning of films and their eventual rupture. At the heart of the model is a separation of the relevant foam physics into different time and length scales which are first treated individually (e.g. using the Navier Stokes equation) and then combined. Further calculations with this model are required to establish how its predictions relate to experimental findings for structure (and other properties) of bulk foam.

\subsection{Analytical techniques}

The availability of X-ray tomography, while currently still mainly restricted to (moderately) wet foams, contributes greatly to the understanding of foams $[4,17,47-49]$. Data includes bubble size distributions for both bulk and surface bubbles and distributions of the number of faces of bubbles, of interest, for example, when studying coarsening due to gas diffusion between bubbles $[17,49]$. Since the tomography results in values for the position of every bubble one can in principle also compute correlations between neighbouring bubbles, similar to the data presented in Section 2 from Surface Evolver calculations [17,49].

As in granular materials $[97,98]$, the presence or absence of order in large samples is of considerable interest, as discussed in Section 3. A number of measures of order are available and can readily be applied to tomographic data. Computation of the radial distribution function $\mathrm{g}(\mathrm{r})$, related to the probability of finding a bubble within a given distance $r$ to a given bubble in the foam, allows for the detection of global ordering within a sample. Bond orientational order parameters probe ordering at a local level [99]. Their computation requires as input the position of all nearest neighbours of a specified bubble. A particular subset of these parameters (called $\mathrm{Q}_{46}$ 


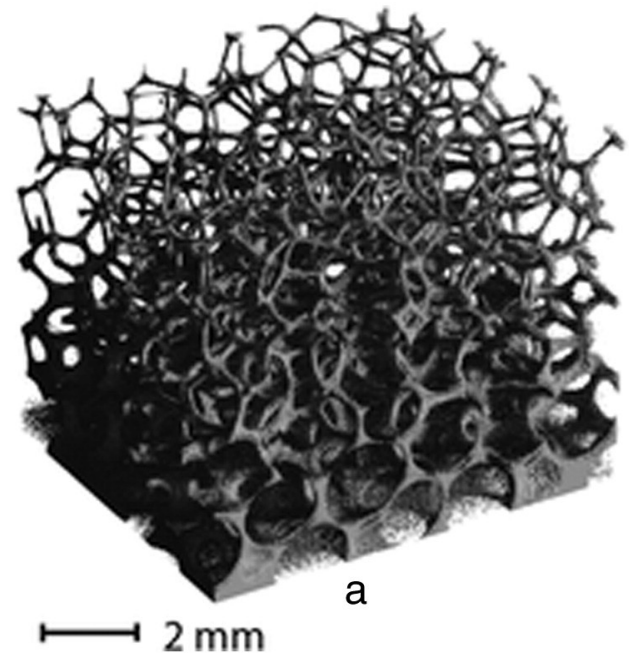

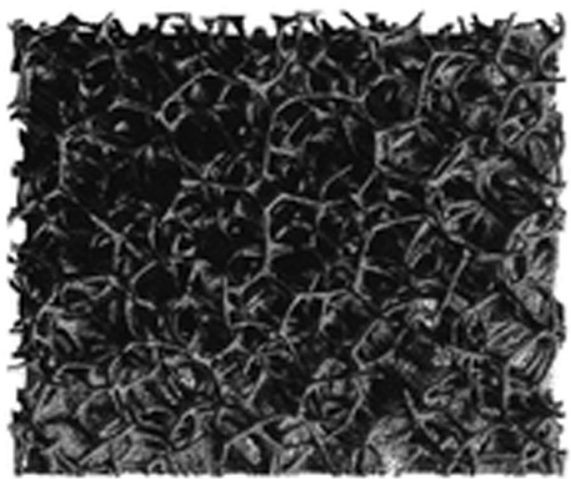

b

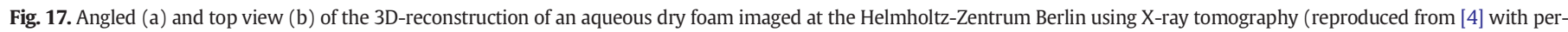
mission from the Royal Society of Chemistry).

[100]) may be used to probe whether the local bubble arrangement is of the ABC type (FCC) or ABA (HCP). Fig. 18 illustrates their application.

\section{Conclusions and outlook}

Foam structure depends on both the method of foam production and (local) liquid fraction. While the production method determines the degree of mono- or polydispersity, and the bubble ordering, the liquid fraction controls the overall bubble shape, ranging from polyhedral for dry foams and near spherical for wet foams.

In the limit of low liquid fraction, the structure of dry foams may be considered well understood (Section 2), thanks to a plethora of experimental results and numerical Surface Evolver data.

In the wet foam limit, the existence of a rigidity loss transition, in analogy to an (un-)jamming transition in granular materials [52-54, $56,101]$, makes it tempting to consider wet foams as packings of soft granular particles with non-pairwise interaction potentials (Section 4). More simulations and experiments, e.g. tomography for the gathering of contact number data, are required.

Important questions remain in the description of foam structure at intermediate values of liquid fractions (Section 4). The concept of a non-entropic osmotic pressure for the description of foam properties is a good approach, but more experiments and simulations are needed.
In particular, important questions remain as to how one can appropriately characterise the structure of foam at intermediate and high liquid fraction.

We have neglected questions of physical chemistry in this article by assuming a constant surface tension throughout the foam. However, a range of "modern" liquid foams employ stabilising agents (such as surface active nano-particles, special proteins or polymers) which lead to viscoelastic interfaces which may influence foam dynamics and therefore foam structure in equilibrium [74]. Particularly relevant might be the influence of static friction between bubbles on the jamming transition, as this is known to play a role in granular matter. Moreover, a finite interfacial elasticity implies an additional energy cost for deforming the bubbles away from their spherical shape. On a macroscopic level, this will increase the osmotic pressure with increasing elasticity and deformation. In turn, this would affect the liquid fraction profile under gravity: the foam will be less drained in equilibrium. On a microscopic level this could, for example, modify Plateaus laws in dry foams.

The combination of our improved understanding of the structure of foam, together with the recently developed novel methods for the solidification of liquid samples [102-106], provides great scope for in-depth investigations of the structure/property relationships of solid foams, and for the development of new foam materials with purposedesigned properties.
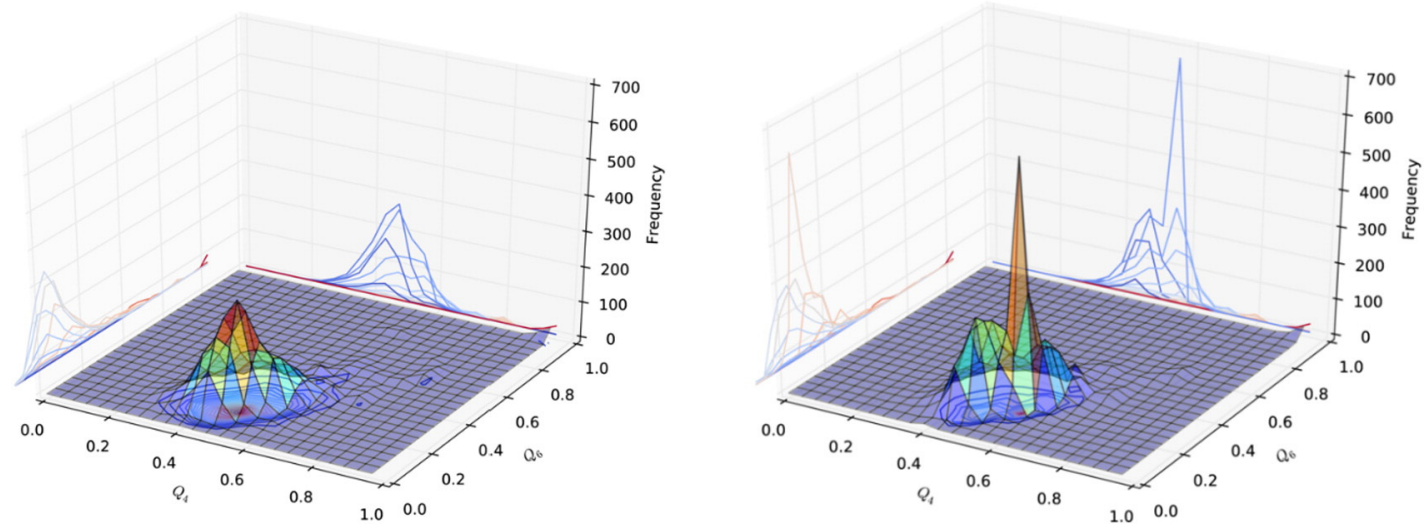

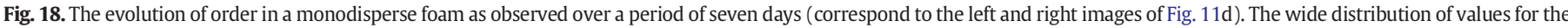

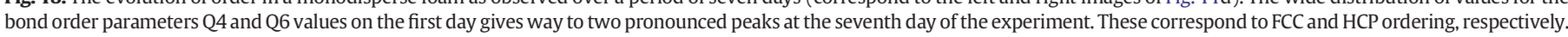
(Reproduced from [25] with permission from the Royal Society of Chemistry.). 


\section{Acknowledgements}

We would like to thank Denis Weaire for critical comments on the manuscript, Simon Cox for providing numerous Surface Evolver images and David Whyte for some computations and graphs. WD thanks R. Höhler, S. Heitkam, E. Rio, A. Salonen and D. Langevin for numerous discussions related to foam structure. WD acknowledges funding from the European Research Council (ERC Starting Grant agreement 307280POMCAPS). SH acknowledges that this publication has emanated from research supported in part by a research grant from Science Foundation Ireland (SFI) under the grant number 13/IA/1926. We acknowledge support of the MPNS COST Actions MP1106 "Smart and green interfaces" and MP1305 "Flowing Matter".

\section{Appendix A. Some structural relations for the Kelvin foam}

While in this article we highlighted the differences between various foam structures, the identification of a model foam structure is beneficial for many theoretical and practical purposes. The Kelvin structure $[1,28]$, introduced in Section 2, has proven useful to estimate some key structural properties of the foam. The simplicity of these calculations results from the fact that all edge lengths of the Kelvin cell have the same length $l$, as shown in Fig. 19.

A Kelvin cell has 8 hexagonal faces, 6 square faces and 36 edges (Plateau borders), each of length 1 . Every cell is associated with 12 Plateau borders.

The surface area $S_{K}$ of a perfectly dry Kelvin foam with flat interfaces $(\varphi=0)$ can be written as

$S_{K}=(12 \sqrt{3}+6) l^{2}$

while for the cell volume one finds

$V_{K}=8 \sqrt{2} l^{3}$

leading to an equivalent bubble radius of

$R_{B}=\left(\frac{6 \sqrt{2}}{\pi}\right) l$

Combining Eqs. (20) and (21) leads to a value of $\hat{E}=$ $3(2 \sqrt{3}+1) 2^{-\frac{4}{3}} \approx 5.315$ for the dimensionless scaled energy density introduced in Eq. (3). This compares with $\hat{E} \approx 5.306$ for the energy minimised Kelvin structure with its curved hexagonal faces.

For a slightly wet Kelvin foam the following relation holds between radius of curvature of the Plateau border $r_{\mathrm{PB}}$ and liquid fraction [28],

$\varphi_{K} \approx 0.17\left(\frac{r_{\mathrm{PB}}}{l}\right)^{2}+0.2\left(\frac{r_{\mathrm{PB}}}{l}\right)^{3}$.

The first and second term account for the liquid in the Plateau borders and their junctions, respectively. For a sufficiently dry foam $\left(r_{\mathrm{PB}}<l \rightarrow \varphi<0.01\right)$ with negligible vertex volume this results in

$\varphi_{K}(\varphi<0.01) \approx 0.33\left(\frac{r_{\mathrm{PB}}}{R_{B}}\right)^{2}$

At $\varphi^{\prime} \approx 0.063$ the FCC structure has a lower energy than the Kelvin structure (see Fig. 12). Around $\varphi \approx 0.11$ the square faces of the Kelvin structure shrink to zero; the structure becomes mechanically unstable.

\section{Appendix B. Osmotic pressure and profiles of liquid fraction}

A column of foam floating on top of the foaming solution (see example in Fig. 1) has a well-defined equilibrium liquid fraction profile $\varphi(h)$, where $h$ is measured upwards from the liquid interface at $h=0$. This profile results from the balance of gravitational forces, which drain the liquid downwards, and capillary forces, which resist the associated bubble deformation.

One may imagine placing a horizontal surface of unit area into this column at some height $h$. The upward force exerted on this surface by the gas phase equals the local osmotic pressure. It is given by the combined buoyancy force of all the bubbles below the surface.

The variation of the osmotic pressure is thus related to the local liquid fraction by

$d \Pi=(1-\varphi(h)) \Delta \rho g d h$,

or in dimensionless form by

$d \tilde{\Pi}=(1-\varphi(\tilde{h})) d \tilde{h}$,

where

$\tilde{h}=R_{32} / l_{c}^{2} h$,

is the normalised vertical positionand $l_{\mathrm{c}}$ the capillary length, which was introduced for Eq. (2). Fig. 20a displays a selection of liquid fraction profiles for different types of foams, obtained from measurements of local electrical conductivity [77]. The strong dependence of the liquid fraction profile on the bubble size is clearly visible. Fig. 20b shows that using the renormalisation of Eq. (27) all liquid fraction profiles fall onto a master curve. Integration of this data using Eq. (26) results in the variation of osmotic pressure with liquid fraction $\Pi(\varphi)$.

Using Höhler's empirical osmotic pressure law of Eq. (18) in the integration of Eq. (26) leads to an explicit approximation of the liquid fraction profile in a foam over the entire range of liquid fraction,

$\tilde{h}=K\left[\left(\sqrt{\phi_{c}}-\sqrt{\phi}\right)\left(3+\frac{\sqrt{\varphi_{c}^{3}}}{\sqrt{\varphi}}\right)+\frac{1}{2}\left(3-2 \varphi_{c}-\varphi_{c}^{2}\right) \ln \left(\frac{(\sqrt{\varphi}+1)\left(\sqrt{\varphi_{c}}-1\right)}{(\sqrt{\varphi}-1)\left(\sqrt{\varphi_{c}}+1\right)}\right)\right]$

with $K=7.3$ and $\varphi_{\mathrm{c}}=0.26$ for monodisperse ordered foams and $K=$ 3.2 and $\varphi_{\mathrm{c}}=0.36$ for disordered foams [77]. The inverse relationship for liquid fraction as a function of vertical position of the foam can only be obtained numerically.
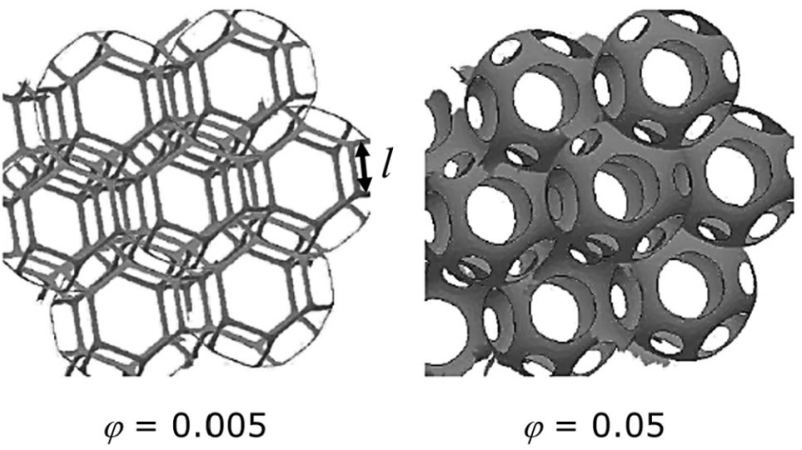

Fig. 19. Surface Evolver computation of a Kelvin foam at two different values of liquid fraction (courtesy of S. Cox, see also [24]). 

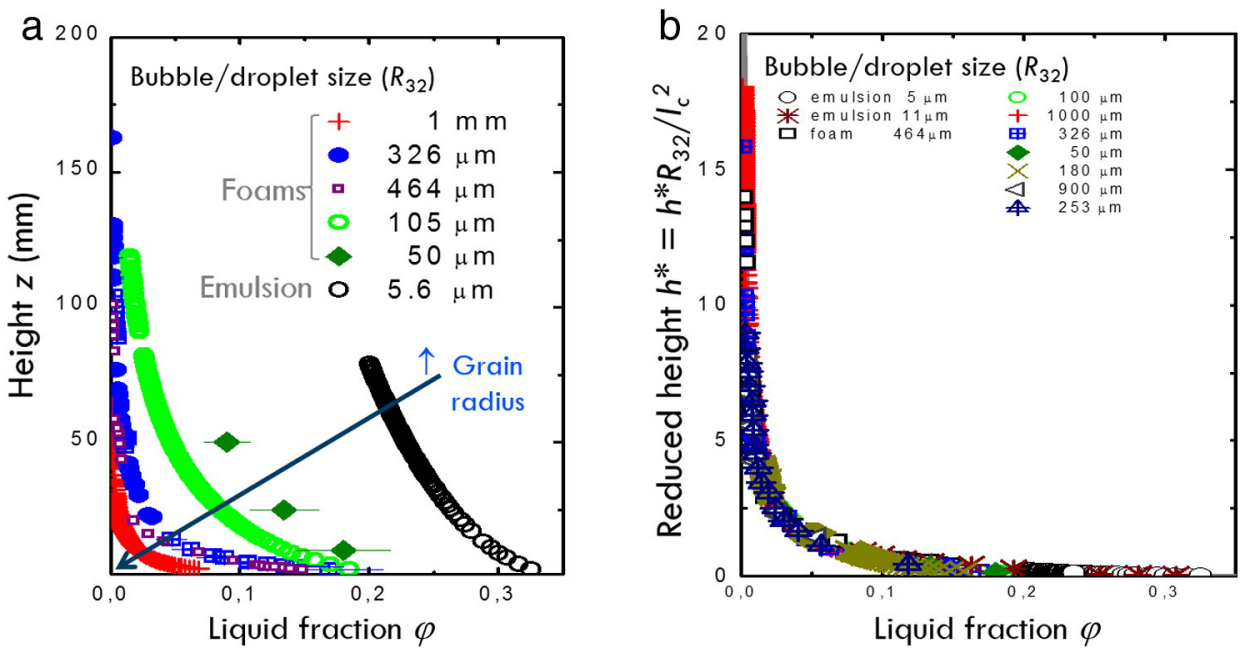

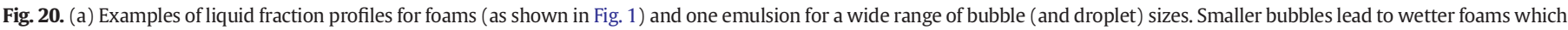
also follows from the Princen number Pri given in Eq. (2). (b) Same data as in (a) after renormalisation of the foam height by $R_{32} / l_{\mathrm{c}}^{2}$ given in Eq. (27). (Data from [77]).

A mathematically simpler expression is given by $[1,76]$

$\varphi(\tilde{h})=\frac{\phi_{c}}{(1+\tilde{h})^{2}}$.

Surprisingly, as shown in Fig. 21, this simple formula compares quite well with experimental data and may be sufficient for many practical purposes.

Expressions for $\varphi(h)$ may also be used to answer Princen's question: what is the volume of liquid $V_{1}$ contained in an infinitely tall column of foam of cross-sectional area $A$ and bubble size $R_{32}$ ? In general terms one can show that [80]

$V_{l}=C_{V} A \frac{l_{c}^{2}}{R_{32}}$

While Krotov estimated $C_{V}$ to be between 0.1 and 0.2 [107] and Princen approximated it as 0.28 [80], Maestro et al. propose that

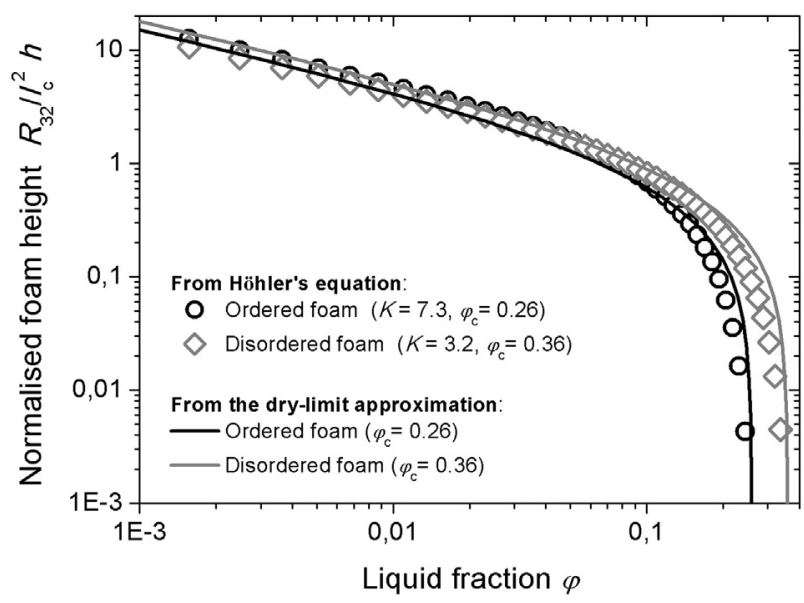

Fig. 21. Liquid fraction profiles obtained for ordered and disordered foams using Maestro's Eq. (28) (based on Höhler's Eq. (18)) and the dry limit approximation of Eq. (29). In both cases, the constants $(K$ or $c$ ) in the expressions were obtained using Princen's criterion (19).
$C_{\mathrm{V}}=0.29$ for monodisperse, ordered and $C_{\mathrm{V}}=0.30$ for polydisperse foams [77]. Using the simple liquid fraction formula of above (Eq. (29)) leads to $C_{V}=0.34$ (for Kelvin) and $C_{V}=0.33$ for random foam.

\section{References}

[1] Weaire D, Hutzler S. The physics of foams. Oxford: Clarendon Press; 1999.

[2] Aste T, Di Matteo T, editors. Granular and complex materials. World Scientific; 2007.

[3] Drenckhan W, Langevin D. Monodisperse foams in one to three dimensions. Curr Opin Colloid Interface Sci 2010;15(5):341-58.

[4] Meagher AJ, Mukherjee M, Weaire D, Hutzler S, Banhart J, Garcia-Moreno F. Analysis of the internal structure of monodisperse liquid foams by X-ray tomography. Soft Matter 2011;7(21):9881-5.

[5] van der Net A, Drenckhan W, Weaire D, Hutzler S. The crystal structure of bubbles in the wet foam limit. Soft Matter 2006;2(2):129-34.

[6] Höhler R, Sang YYC, Lorenceau E, Cohen-Addad S. Osmotic pressure and structures of monodisperse ordered foam. Langmuir 2007;24(2):418-25.

[7] van der Net A, Delaney GW, Drenckhan W, Weaire D, Hutzler S. Crystalline arrangements of microbubbles in monodisperse foams. Colloids Surf A Physicochem Eng Asp 2007:309(1-3):117-24.

[8] Gabbrielli R, Meagher AJ, Weaire D, Brakke KA, Hutzler S. An experimental realization of the Weaire-Phelan structure in monodisperse liquid foam. Philos Mag Lett 2012;92(1):1-6.

[9] Meagher AJ, McAteer D, Hutzler S, Weaire D. Building the pyramids: perfect bubble crystals. Philos Mag 2013;93(31-33):4138-50.

[10] Saadatfar M, Barry J, Weaire D, Hutzler S. Ordered cylindrical foam structures with internal bubbles. Philos Mag Lett 2008;88(9):661-8.

[11] Pittet N, Rivier N, Weaire D. Cylindrical packing of foam cells. Forma 1995;10(1): 65-73.

[12] Meagher AJ, García-Moreno F, Banhart J, Mughal A, Hutzler S. An experimental study of columnar crystals using monodisperse microbubbles. Colloids Surf A Physicochem Eng Asp 2015;473:55-9.

[13] Hutzler S, Barry J, Grasland-Mongrain P, Smyth D, Weaire D. Ordered packings of bubbles in columns of square cross-section. Colloids Surf A Physicochem Eng Asp 2009;344(1-3):37-41.

[14] Drenckhan W, Weaire D, Cox SJ. The demonstration of conformal maps with twodimensional foams. Eur J Phys 2004;25(3):429-38.

[15] Quilliet C, Talebi SA, Rabaud D, Kafer J, Cox SJ, Graner F. Topological and geometrical disorders correlate robustly in two-dimensional foams. Philos Mag Lett 2008; 88(9-10):651-60.

[16] Durand M, Kraynik AM, van Swol F, Kafer J, Quilliet C, Cox S, et al. Statistical mechanics of two-dimensional shuffled foams: geometry-topology correlation in small or large disorder limits. Phys Rev E 2014;89(6).

[17] Lambert J, Mokso R, Cantat I, Cloetens P, Glazier JA, Graner F, et al. Coarsening foams robustly reach a self-similar growth regime. Phys Rev Lett 2010;104(24).

[18] Fortuna I, Thomas GL, de Almeida RMC, Graner F. Growth laws and self-similar growth regimes of coarsening two-dimensional foams: transition from dry to wet limits. Phys Rev Lett 2012;108(24):248301.

[19] de Almeida RMC, Thomas GL, Graner F. Universal, statistically scale-invariant regime in 3D cellular systems. Phys A: Stat Mech Appl 2006;371(1):67-70.

[20] Cox SJ, Graner F. Three-dimensional bubble clusters: shape, packing, and growth rate. Phys Rev E 2004;69(3):031409. 
[21] Drenckhan W, Saint-Jalmes A. The science of foaming. Adv Colloid Interf Sci 2015. http://dx.doi.org/10.1016/j.cis.2015.04.001.

[22] Kraynik AM, Reinelt DA, van Swol F. Structure of random foam. Phys Rev Lett 2004; 93(20):208301-4

[23] Kraynik AM. The structure of random foam. Adv Eng Mater 2006;8(9):900-6.

[24] Cox S, Weaire D, Brakke K. Liquid foams - precursors for solid foams. In: Scheffler PCaM, editor. Cellular ceramics: structure, manufacturing, properties and applications. Wiley; 2005.

[25] Meagher AJ, Whyte D, Banhart J, Weaire D, Hutzler S, Garcia-Moreno F. Slow crystallisation of a monodisperse foam stabilised against coarsening. Soft Matter 2015;11:4710-6. http://dx.doi.org/10.1039/C4SM02412E [p.].

[26] Heitkam S, Drenckhan W, Fröhlich J. Packing spheres tightly: influence of mechanical stability on close-packed sphere structures. Phys Rev Lett 2012;108(14): 148302.

[27] Plateau JAF. Statique Expérimentale et Théorieque des Liquides soumis aux seules Forces Moléculaires. , 2Paris: Gauthier-Villars; 1873.

[28] Cantat I, Cohen-Addad S, Elias F, Graner F, Höhler R, Pitois O, et al. Foams - structure and dynamics. In: Cox S, editor. Oxford, UK: Oxford University Press: 2013, p. 300.

[29] Brakke K. Instability of the wet cube cone soap film. Colloids Surf A Physicochem Eng Asp 2005;263(1-3):4-10.

[30] Besson S, Debregeas G. Statics and dynamics of adhesion between two soap bubbles. Eur Phys J E 2007:24(2):109-17.

[31] Fortes MA, Teixeira PIC. Triple-line decoration and line tension in simple threedimensional foam clusters. Phys Rev E 2005;71(5).

[32] Jurine S, Cox S, Graner F. Dry three-dimensional bubbles: growth-rate, scaling state and correlations. Colloids Surf A Physicochem Eng Asp 2005;263(1-3):18-26.

[33] Brakke K. The Surface Evolver. Exp Math 1992;1(2):141-65.

[34] Weaire D, editor. The Kelvin Problem. CRC Press; 1997.

[35] Weaire D, Phelan R. A counterexample to Kelvin's conjecture on minimal surfaces. Philos Mag Lett 1994;69:107-10.

[36] Kraynik AM, Reinelt DA, van Swol F. Structure of random monodisperse foam. Phys Rev E 2003;67(3):031403.

[37] Gabbrielli R. A new counter-example to Kelvin's conjecture on minimal surfaces. Philos Mag Lett 2009;89(8):483-91.

[39] Kusner R. The number of faces in a minimal foam. Proc R Soc Lond Ser A Math Phys Eng Sci 1992;439(1907):683-6.

[40] Sullivan JM. The geometry of bubbles and foams. In: Rivier N, Sadoc JF, editors. Foams and Emulsions. Dordrecht: Kluwer; 1999. p. 403.

[41] Matzke EB. The three-dimensional shape of bubbles in foam-an analysis of the role of surface forces in three-dimensional cell shape determination. Am J Bot 1946 ; 33(1):58-80.

[42] Kraynik AM, Reinelt DA, van Swol F. Structure of random bidisperse foam. Colloids Surf A Physicochem Eng Asp 2005;263(1-3):11-7.

[43] Newhall KA, Pontani LL, Jorjadze I, Hilgenfeldt S, Brujic J. Size-topology relations in packings of grains, emulsions, foams, and biological cells. Phys Rev Lett 2012; 108(26).

[44] Wang H, Liu GQ. Generalization of the Aboav-Weaire law. EPL (Europhys Lett) 2012;100(6):68001.

[45] Hilgenfeldt S, Kraynik AM, Reinelt DA, Sullivan JM. The structure of foam cells: isotropic plateau polyhedra. Europhys Lett 2004;67(3):484-90.

[46] Cox SJ, Fortes MA. Properties of three-dimensional bubbles of constant mean curvature. Philos Mag Lett 2003;83(4):281-7.

[47] Mader K, Mokso R, Raufaste C, Dollet B, Santucci S, Lambert J, et al. Quantitative 3D characterization of cellular materials: Segmentation and morphology of foam. Colloids Surf A 2012;415:230-8.

[48] Lambert J, Mokso R, Cantat I, Cloetens P, Glazier JA, Graner F, et al. Coarsening foams robustly reach a self-similar growth regime. Phys Rev Lett 2010:104:248-304.

[49] Lambert J, Cantat I, Delannay R, Mokso R, Cloetens P, Glazier JA, et al. Experimental growth law for bubbles in a moderately "wet" 3D liquid foam. Phys Rev Lett 2007; 99:058304.

[50] Langevin D, Vignes-Adler M. Microgravity studies of aqueous wet foams. Eur Phys ] E 2014;37(3)

[51] O'Hern CS, Silbert LE, Liu AJ, Nagel SR. Jamming at zero temperature and zero applied stress: the epitome of disorder. Phys Rev E 2003;68(1).

[52] van Hecke M. Jamming of soft particles: geometry, mechanics, scaling and isostaticity. J Phys Condens Matter 2010;22(3).

[53] Song C, Wang P, Makse HA. A phase diagram for jammed matter. Nature 2008; 453(7195):629-32.

[54] Lespiat R, Cohen-Addad S, Höhler R. Jamming and flow of random close packed spherical bubbles: an analogy with granular materials. Phys Rev Lett 2011;106: 148302.

[55] Farr RS, Groot RD. Close packing density of polydisperse hard spheres. J Chem Phys 2009;131(24)

[56] Katgert G, Tighe BP, van Hecke M. The jamming perspective on wet foams. Soft Matter 2013:9(41):9739-46.

[57] Jorjadze I, Pontani L-L, Brujic J. Microscopic approach to the nonlinear elasticity of compressed emulsions. Phys Rev Lett 2013;110(4).

[58] Corwin EI, Clusel M, Siemens AON, Brujic J. Model for random packing of polydisperse frictionless spheres. Soft Matter 2010;6(13):2949-59.

[59] Katgert G, van Hecke M. Jamming and geometry of two-dimensional foams. EPL 2010;92(3).

[60] Delaney G, Weaire D, Hutzler S, Murphy S. Random packing of elliptical disks. Philos Mag Lett 2005;85(2):89-96.

[61] Bragg L, Nye JF. A dynamical model of a crystal structure. Proc R Soc Lond Ser A Math Phys Sci 1947;190(1023):474-81.

[62] Hales TC. A proof of the Kepler conjecture. Ann Math 2005;162:1065-85.
63] Kempe T, Froehlich J. An improved immersed boundary method with direct forcing for the simulation of particle laden flows. J Comput Phys 2012; 231(9):3663-84

64] Heitkam S. Manipulation of liquid metal foam with electromagnetic fields: a numerical study, PhD thesis. Technische Universitaet Dresden \& Université ParisSud; 2014

[65] Barrett DGT, Kelly S, Daly EJ, Dolan MJ, Drenckhan W, Weaire D, et al. Taking Plateau into microgravity: the formation of an eightfold vertex in a system of soap films. Microgravity Sci Technol 2008;20(1):17-22.

[66] Weaire D. Structural transformations in foam. Philos Mag Lett 1994;69(2):99-105.

[67] Weaire D, Vaz MF, Teixeira PIC, Fortes MA. Instabilities in liquid foams. Soft Matter 2007:3(1):47-57.

[68] Mason TG, Lacasse M-D, Grest GS, Levine D, Bibette J, Weitz DA. Osmotic pressure and viscoelastic shear moduli of concentrated emulsions. Phys Rev E 1997;56(3):3150.

[69] Mason TG, Bibette J, Weitz DA. Elasticity of compressed emulsions. Phys Rev Lett 1995;75(10):2051.

[70] Lacasse M-D, Grest GS, Levine D. Deformation of small compressed droplets. Phys Rev E 1996:54(5):5436.

[71] Durian DJ. Foam mechanics at the bubble scale. PRL 1995:75(26):4780-3.

[72] Saint-Jalmes A, Durian DJ. Vanishing elasticity for wet foams: equivalence with emulsions and role of polydispersity. J Rheol 1999;43(6):1411-22.

[73] Durian DJ. Bubble-scale model of foam mechanics: melting, nonlinear behavior, and avalanches. Phys Rev E 1997;55(2):1739-51.

[74] Cohen-Addad S, Höhler R, Pitois O. Flow in foams and flowing foams. Annu Rev Fluid Mech 2013:45:241-67.

[75] Hutzler S, Murtagh R, Whyte D, Tobin ST, Weaire D. Z-cone model for the energy of an ordered foam. Soft Matter 2014;10:7103-8

[76] Whyte D, Murtagh R, Weaire D, Hutzler S. Applications and extensions of the Zcone model for the energy of a foam. Colloids Surf A Physicochem Eng Asp 2015; 473:115-22.

[77] Maestro A, Drenckhan W, Rio E, Höhler R. Liquid dispersions under gravity: volume fraction profile and osmotic pressure. Soft Matter 2013:9(8):2531-40.

[78] Princen HM. The structure, mechanics, and rheology of concentrated emulsions and fluid foams. In: Sjoblom J, editor. Encyclopedic handbook of emulsion technology. Marcel Dekker; 2000. p. 243.

[79] Princen HM, Kiss AD. Osmotic-pressure of foams and highly concentrated emulsions. 2. Determination from the variation in volume fraction with height in an equilibrated column. Langmuir 1987;3(1):36-41.

[80] Princen HM. Osmotic pressure of foams and highly concentrated emulsions. I. Theoretical considerations. Langmuir 1986:2(4):519-24.

[81] Feitosa K, Marze S, Saint-Jalmes A, Durian DJ. Electrical conductivity of dispersions: from dry foams to dilute suspensions. J Phys Condens Matter 2005;17(41):6301-5.

[82] Solórzano E, Pardo-Alonso S, de Saja JA, Rodríguez-Pérez MA. Study of aqueous foams evolution by means of X-ray radioscopy. Colloids Surf A Physicochem Eng Asp 2013;438:159-66

83] Garcia-Moreno F, Tobin ST, Mukherjee M, Jimenez C, Solorzano E, Vinod Kumar GS, et al. Analysis of liquid metal foams through X-ray radioscopy and microgravity experiments. Soft Matter 2014;10(36):6955-62.

[84] Wang YJ, Neethling SJ. The relationship between the surface and internal structure of dry foam. Colloids Surf A Physicochem Eng Asp 2009;339(1-3):73-81.

[85] Cheng HC, Lemlich R. Errors in the measurement of bubble size distribution in foam. Ind Eng Chem Fundam 1983;22(1):105-9.

[86] Gaillard T, Honorez C, Jumeau M, Elias F, Drenckhan W. A simple technique for the automation of bubble size measurements. Colloids Surf A 2015:473:68-74.

[87] Vera MU, Saint-Jalmes A, Durian DJ. Scattering optics of foam. Appl Opt 2001; 40(24):4210-4.

[88] van der Net A, Blondel L, Saugey A, Drenckhan W. Simulating and interpreting images of foams with computational ray-tracing techniques. Colloids Surf A Physicochem Eng Asp 2007;309(1-3):159-76.

[89] Clusel M, Corwin EI, Siemens AON, Brujic J. A 'granocentric' model for random packing of jammed emulsions. Nature 2009;460:611-6.

[90] Monnereau C, Prunet-Foch B, Vignes-Adler M. Topology of slightly polydisperse real foams. Phys Rev E 2001;63(6).

[91] Fetterman MR, Tan E, Ying L, Stack RA, Marks DL, Feller S, et al. Tomographic imaging of foam. Opt Express 2000;7(5):186-97.

[92] Monnereau C, Vignes-Adler M. Optical tomography of real three-dimensional foams. J Colloid Interface Sci 1998;202:45-53.

[93] Prause BA, Glazier JA, Gravina SJ, Montemagno CD. 3-Dimensional magneticresonance-imaging of a liquid foam. J Phys Condens Matter 1995;7(40):L511-6.

[94] Jiang Y, Glazier JA. Extended large-Q Potts model simulation of foam drainage. Philos Mag Lett 1996;74(2):119-28.

[95] Thomas GL, de Almeida RMC, Graner F. Coarsening of three-dimensional grains in crystals, or bubbles in dry foams, tends towards a universal, statistically scaleinvariant regime. Phys Rev E Stat Nonlinear Soft Matter Phys 2006;74(2): 021407-18.

[96] Saye RI, Sethian JA. Multiscale modeling of membrane rearrangement, drainage, and rupture in evolving foams. Science 2013;340(6133):720-4.

[97] Torquato S, Truskett TM, Debenedetti PG. Is random close packing of spheres well defined? Phys Rev Lett 2000;84(10):2064-7.

[98] Torquato S. Random heterogeneous materials: microstructure and macroscopic properties. Springer Science \& Business Media; 2002

[99] Steinhardt PJ, Nelson DR, Ronchetti M. Bond-orientational order in liquids and glasses. Phys Rev B 1983;28(2):784-805.

[100] Mickel W, Kapfer SC, Schröder-Turk GE, Mecke K. Shortcomings of the bond orientational order parameters for the analysis of disordered particulate matter. J Chem Phys 2013;138(4):044501. 
[101] Ikeda A, Berthier L, Sollich P. Unified study of glass and jamming rheology in soft particle systems. Phys Rev Lett 2012;109(1):018301.

[102] Testouri A, Honorez C, Barillec A, Langevin D, Drenckhan W. Highly structured foams from chitosan gels. Macromolecules 2010;43(14):6166-73.

[103] Testouri A, Arriaga LR, Honorez C, Ranft M, Rodrigues J, van der Net A, et al. Generation of porous solids with well-controlled morphologies by combining foaming and flow chemistry on a Lab-on-a-Chip. Colloids Surf A Physicochem Eng Asp 2012;413:17-24.

[104] Testouri A, Ranft M, Honorez C, Kaabeche N, Ferbitz J, Freidank D, et al. Generation of crystalline polyurethane foams using millifluidic lab-on-a-chip technologies. Adv Eng Mater 2013;15(11):1086-98.

[105] van der Net A, Gryson A, Ranft M, Elias F, Stubenrauch C, Drenckhan W. Highly structured porous solids from liquid foam templates. Colloids Surf A Physicochem Eng Asp 2009;346(1-3):5-10.
[106] Kraynik AM. Foam structure: from soap froth to solid foams. MRS Bull 2003; 28(04):275-8.

[107] Krotov VV. Theory of syneresis of foams and concentrated emulsions. 1. Local aeration ratio in polyhedral disperse systems Colloid J USSR 1980;42:903.

[108] Garrett PR, Hines JD, Joyce SC, Whittal PT. Report prepared for Univlever R\&amp;D Port Sunlight; 1993.

[109] Mukherjee S, Wiedersich H. Morphological and viscoelastic properties of dense foams generated from skin cleansing bars. Colloids Surf A Physicochem Eng Asp 1995;95(2-3):159-72.

[110] Thomson W. On the division of space with minimum partitional area (Reprinted) Philos Mag Lett 2008;88(2):A503-14. 